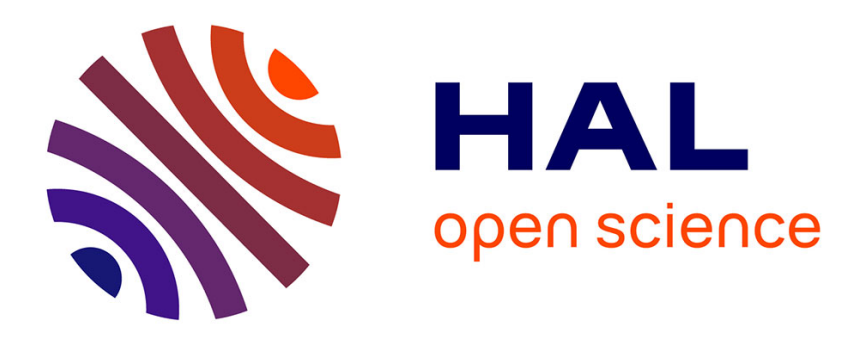

\title{
Elliptic double shuffle, Grothendieck-Teichmüller and mould theory
}

Leila Schneps

\section{To cite this version:}

Leila Schneps. Elliptic double shuffle, Grothendieck-Teichmüller and mould theory. Annales mathématiques du Québec, 2020. hal-02994272

\section{HAL Id: hal-02994272 \\ https://hal.science/hal-02994272}

Submitted on 7 Nov 2020

HAL is a multi-disciplinary open access archive for the deposit and dissemination of scientific research documents, whether they are published or not. The documents may come from teaching and research institutions in France or abroad, or from public or private research centers.
L'archive ouverte pluridisciplinaire HAL, est destinée au dépôt et à la diffusion de documents scientifiques de niveau recherche, publiés ou non, émanant des établissements d'enseignement et de recherche français ou étrangers, des laboratoires publics ou privés. 


\title{
Elliptic double shuffle, Grothendieck-Teichmüller and mould theory
}

\author{
Leila Schneps
}

\begin{abstract}
In this article we define an elliptic double shuffle Lie algebra $\mathfrak{d} \mathfrak{s}_{\text {ell }}$ that generalizes the well-known double shuffle Lie algebra $\mathfrak{d s}$ to the elliptic situation. The double shuffle, or dimorphic, relations satisfied by elements of the Lie algebra $\mathfrak{d} \mathfrak{s}$ express two families of algebraic relations between multiple zeta values that conjecturally generate all relations. In analogy with this, elements of the elliptic double shuffle Lie algebra $\mathfrak{d} \mathfrak{s}_{e l l}$ are Lie polynomials having a dimorphic property called $\Delta$-bialternality that conjecturally describes the (dual of the) set of algebraic relations between elliptic multiple zeta values, which arise as coefficients of a certain elliptic generating series (constructed explicitly in [LMS]) and closely related to the elliptic associator defined by Enriquez ([En1]). We show that one of Ecalle's major results in mould theory can be reinterpreted as yielding the existence of an injective Lie algebra morphism $\mathfrak{d} \mathfrak{s} \rightarrow \mathfrak{d} \mathfrak{s}_{\text {ell }}$. Our main result is the compatibility of this map with the tangential-base-point section Lie $\pi_{1}(M T M) \rightarrow$ Lie $\pi_{1}(M E M)$ constructed by Hain and Matsumoto and with the section $\mathfrak{g r t} \rightarrow \mathfrak{g r t} \mathfrak{t}_{\text {ell }}$ mapping the Grothendieck-Teichmüller Lie algebra grt into the elliptic Grothendieck-Teichmüller Lie algebra $\mathfrak{g r t}_{\text {ell }}$ constructed by Enriquez. This compatibility is expressed by the commutativity of the following diagram (excluding the dotted arrow, which is conjectural).
\end{abstract}

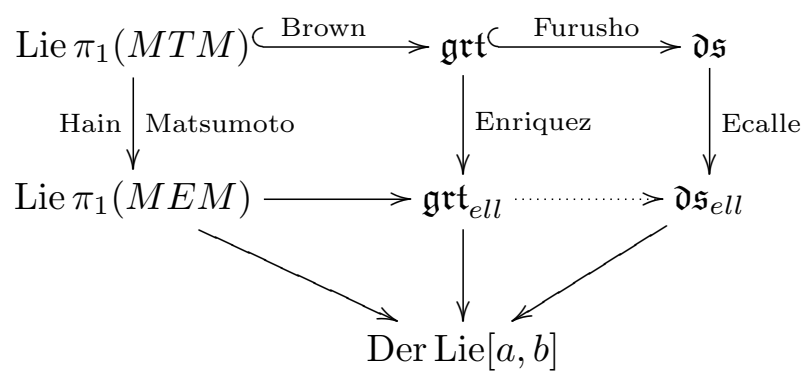

Résumé. Dans cet article, nous définissons une algèbre de Lie de double mélange elliptique das $\mathfrak{s l l}$ qui généralise l'algèbre de Lie bien connue de double mélange ds au cas elliptique. Les relations de double mélange (ou dimorphiques) satisfaites par les éléments de l'algèbre de Lie da expriment deux familles de relations algébriques entre les valeurs zêta multiples, qui engendrent conjecturalement toutes les relations. En analogie avec cette conjecture, les éléments de l'algèbre de double mélange elliptique $\mathfrak{d} \mathfrak{s}_{\text {ell }}$ sont des polynômes de Lie ayant une propriété dimorphique, appelée $\Delta$-bialternalité, qui décrit conjecturalement (le dual de) l'ensemble des relations algébriques entre les valeurs zêtas elliptiques multiples, qui sont les coefficients d'une certaine série génératrice elliptique (construite explicitement dans [LMS]) reliée à l'associateur d'Enriquez ([En1]). Nous montrons que l'un des résultats majeurs de la théorie des moules de J. Écalle peut être interprété comme l'existence d'un morphisme injectif $\mathfrak{d} \mathfrak{s} \rightarrow \mathfrak{d} \mathfrak{s}_{e l l}$ d'algèbres de Lie. Notre résultat principal est la compatibilité de ce morphisme avec la section "point base tangentiel" Lie $\pi_{1}(M T M) \rightarrow$ Lie $\pi_{1}(M E M)$ construite par Hain and Matsumoto ([HM]), et avec la section $\mathfrak{g r t} \rightarrow \mathfrak{g r t} \mathfrak{t}_{e l l}$ construite par Enriquez qui envoie l'algèbre de Lie grt de Grothendieck-Teichmüller vers sa version elliptique $\mathfrak{g r t}_{\text {ell }}$. Ces compatibilités sont exprimées par la commutativité du diagramme (A) (à l'exception de la flèche en pointillé, qui est conjecturale.)

2010 Mathematics Subject Classification: 11M32 


\section{Introduction}

\subsection{Overview}

The goal of this paper is to apply Ecalle's mould theory to define an elliptic double shuffle Lie algebra $\mathfrak{d} \mathfrak{s}_{\text {ell }}$ that turns out to parallel Enriquez' construction in [En1] of the elliptic GrothendieckTeichmüller Lie algebra, and Hain and Matsumoto's construction of the fundamental Lie algebra of the category $M E M$ of mixed elliptic motives in [HM]. Both of those Lie algebras are equipped with canonical surjections to the corresponding genus zero Lie algebras,

$$
\left\{\begin{array}{l}
\mathfrak{g r t}_{\text {ell }} \rightarrow \mathfrak{g r t} \\
\operatorname{Lie} \pi_{1}(M E M) \rightarrow \operatorname{Lie} \pi_{1}(M T M) .
\end{array}\right.
$$

Here, $M T M$ is the category of mixed Tate motives over $\mathbb{Z}$, and the notation Lie $\pi_{1}(M T M)$ (resp. Lie $\pi_{1}(M E M)$ ) denotes the Lie algebra of the pro-unipotent radical of the fundamental group of the Tannakian category $M T M$ (resp. $M E M$ ) equipped with the de Rham fiber functor (resp. its lift to a fiber functor on $M E M$ via composition with the natural surjection $M E M \rightarrow M T M$, cf. $[\mathrm{HM}, \S 5]$.

Each of the Lie algebras $\mathfrak{g r t}_{\text {ell }}$ and Lie $\pi_{1}(M E M)$ is also equipped with a natural section of the above surjection, corresponding, geometrically, to the tangential base point at infinity on the moduli space of elliptic curves:

$$
\left\{\begin{array}{l}
\gamma: \mathfrak{g r t} \hookrightarrow \mathfrak{g r t}_{\text {ell }} \\
\gamma_{t}: \operatorname{Lie} \pi_{1}(M T M) \hookrightarrow \operatorname{Lie} \pi_{1}(M E M) .
\end{array}\right.
$$

Hain-Matsumoto determine a canonical Lie ideal of $\mathfrak{u}$ of Lie $\pi_{1}(M E M)$, and Enriquez defines a canonical Lie ideal $\mathfrak{r}_{\text {ell }}$ of $\mathfrak{g r t}_{\text {ell }}$, such that the above sections give semi-direct product structures

$$
\left\{\begin{array}{l}
\mathfrak{g r t}_{\text {ell }} \simeq \mathfrak{r}_{\text {ell }} \rtimes \gamma(\mathfrak{g r t}) \\
\operatorname{Lie} \pi_{1}(M E M) \simeq \mathfrak{u} \rtimes \gamma_{t}\left(\operatorname{Lie} \pi_{1}(M T M)\right) .
\end{array}\right.
$$

Let $C_{i}=a d(a)^{i-1}(b)$ for $i \geq 1$, and let $\operatorname{Lie}[C]$ denote the Lie algebra Lie $\left[C_{1}, C_{2}, \ldots\right]$. It is an easy consequence of Lazard elimination that Lie $[C]$ is a free Lie algebra on the generators $C_{i}$, and that

$$
\operatorname{Lie}[a, b] \simeq \mathbb{Q} a \oplus \operatorname{Lie}[C]
$$

(see Appendix). In other words, the elements in $\operatorname{Lie}[C]$ are all the elements of $\operatorname{Lie}[a, b]$ having no linear term in $a$.

Definition. Let $\operatorname{Der}^{0} \operatorname{Lie}[a, b]$ denote the subspace of derivations $D \in \operatorname{Der} \operatorname{Lie}[a, b]$ that annihilate $[a, b]$ and such that $D(a)$ and $D(b)$ lie in Lie $[C]$.

Hain-Matsumoto and Enriquez both give derivation representations of the elliptic spaces into $\operatorname{Der}^{0} \operatorname{Lie}[a, b]$, but Enriquez's Lie morphism $\mathfrak{g r t}_{\text {ell }} \rightarrow \operatorname{Der}^{0}$ Lie $[a, b]$ is injective (by [T2], cf. below for more detail), whereas Hain-Matsumoto conjecture this result in the motivic situation. However, Hain-Matsumoto compute the image of $\mathfrak{u}$ in $\operatorname{Der}^{0} \operatorname{Lie}[a, b]$ and show that it is equal to a certain explicitly determined Lie algebra $\mathfrak{b}_{3}$ related to $\mathrm{SL}_{2}\left(\mathbb{Z}\right.$ ) (or to the Artin braid group $B_{3}$ on three strands), namely the Lie algebra generated by derivations $\epsilon_{2 i}, i \geq 0$ defined by $\epsilon_{2 i}(a)=a d(a)^{2 i}(b)$, $\epsilon_{2 i}([a, b])=0^{1}$, whereas Enriquez considers the same Lie algebra $\mathfrak{b}_{3}$, shows that it injects into $\mathfrak{r}_{e l l}$, and conjectures that they are equal ${ }^{2}$.

\footnotetext{
1 This Lie algebra was introduced by Tsunogai in [T1, §3] (see also [P], [BS] and [Br4] for some results on its interesting structure. The $\epsilon_{2 i}$ also play an important role in [CEE] and [En1].

2 It is really remarkable that these two papers were written totally independently of one another.
} 
All these maps are compatible with the canonical injective morphism Lie $\pi_{1}(M T M) \rightarrow \mathfrak{g r t}$ whose existence was proven by Goncharov and Brown in two stages as follows. Goncharov constructed a Hopf algebra $\mathcal{A}$ of motivic zeta values as motivic iterated integrals [G, $\S 5]$, and identified it with a subalgebra of the Hopf algebra of framed mixed Tate motives [G, $\S 8]$; he showed that these motivic zeta values satisfy the associator relations. Brown [Br1] subsequently lifted Goncharov's construction to an algebra $\mathcal{H}$ in which the motivic $\zeta^{m}(2)$ is non-zero, such that in fact $\mathcal{H} \simeq \mathcal{A} \otimes \mathbb{Q}\left[\zeta^{m}(2)\right]$. He was able to compute the structure and the dimensions of the graded parts of $\mathcal{H}$ and thus of $\mathcal{A}$, from which it follows that $\mathcal{A}$ is in fact equal to the full Hopf algebra of framed mixed Tate motives. In the dual situation, this means that the fundamental Lie algebra of $M T M$ injects into the Lie algebra of associators, namely the top arrow of the following commutative diagram:

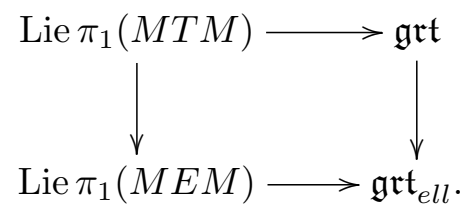

The elliptic double shuffle Lie algebra $\mathfrak{d} \mathfrak{s}_{\text {ell }}$ that we define in this article is conjecturally isomorphic to Lie $\pi_{1}(M E M)$ and $\mathfrak{g r t}_{\text {ell }}$. We show that it shares with them the following properties: firstly, it comes equipped with an injective Lie algebra morphism

$$
\gamma_{s}: \mathfrak{d} \mathfrak{s} \rightarrow \mathfrak{d} \mathfrak{s}_{\text {ell }},
$$

where $\mathfrak{d} \mathfrak{s}$ is the regularized double shuffle Lie algebra defined in $[R]$, where it is denoted $\mathfrak{d} \mathfrak{m} \mathfrak{r}$ ("double mélange régularisé").

Secondly there is an injective derivation representation

$$
\mathfrak{d} \mathfrak{s}_{e l l} \hookrightarrow \operatorname{Der}^{0} \operatorname{Lie}[a, b] .
$$

Unfortunately, we have not yet been able to find a good canonical Lie ideal in $\mathfrak{d} \mathfrak{s}_{\text {ell }}$ that would play the role of $\mathfrak{u}$ and $\mathfrak{r}_{\text {ell }}$, although it is easy to show that there is an injection $\mathfrak{b}_{3} \hookrightarrow \mathfrak{d} \mathfrak{s}_{\text {ell }}$ whose image conjecturally plays this role (cf. the end of section 1.3). Since $\mathfrak{u} \rightarrow \mathfrak{b}_{3} \hookrightarrow \mathfrak{d s}_{\text {ell }}$, we do have a Lie algebra injection,

$$
\text { Lie } \pi_{1}(M E M) \hookrightarrow \mathfrak{d s}_{\text {ell }},
$$

but not the desired injection

$$
\mathfrak{g r t}_{\text {ell }} \hookrightarrow \mathfrak{d} \mathfrak{s}_{\text {ell }},
$$

(the dotted arrow in the diagram in the abstract), which would follow as a consequence of Enriquez' conjecture that $\mathfrak{r}_{\text {ell }}=\mathfrak{b}_{3}$. It would have been nice to give a direct proof of the existence of a Lie algebra morphism $\mathfrak{g r t}_{\text {ell }} \rightarrow \mathfrak{d} \mathfrak{s}_{\text {ell }}$ even without proving Enriquez' conjecture, but we were not able to find one. This result appears like an elliptic version of Furusho's injection $\mathfrak{g r t} \hookrightarrow \mathfrak{d} \mathfrak{s}$ (cf. [F]), and may possibly necessitate some similar techniques.

Our main result, however, is the commutation of the diagram given in the abstract, which does not actually require an injective map $\mathfrak{g r t}_{\text {ell }} \rightarrow \mathfrak{d} \mathfrak{s}_{\text {ell }}$, but, given all the observations above, comes down to the commutativity of the triangle diagram

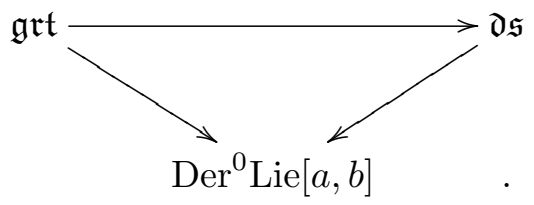


The morphisms from $\mathfrak{g r t}$ and $\mathfrak{d s}$ to Der Lie $[a, b]$ factor through the respective elliptic Lie algebras (cf. the diagram in the abstract). Note that the morphisms in (1.1.1) must not be confused with the familiar Ihara-type morphism $\mathfrak{g r t} \rightarrow$ Der Lie $[x, y]$ via $y \mapsto[\psi(-x-y, y), y]$ and $x+y \mapsto 0$, and the analogous map for $\mathfrak{d} \mathfrak{s}$ investigated in [S2]. The relation between the two is based on the fact that $\operatorname{Lie}[x, y]$ is identified with the Lie algebra of the fundamental group of the thrice-punctured sphere, whereas Lie $[a, b]$ is identified with the Lie algebra of the once-punctured torus. The natural Lie morphism Lie $[x, y] \rightarrow \operatorname{Lie}[a, b]$, reflecting the underlying topology, is given by

$$
x \mapsto t_{01}, y \mapsto t_{02},
$$

where we write $\operatorname{Ber}_{x}=a d(x) /(\exp (a d(x))-1)$ for any $x \in \operatorname{Lie}[a, b]$, and set

$$
t_{01}=\operatorname{Ber}_{b}(-a), \quad t_{02}=\operatorname{Ber}_{-b}(a) .
$$

We show that certain derivations of Lie $[x, y]$, transported to the free Lie subalgebra Lie $\left[t_{01}, t_{02}\right] \subset$ $\operatorname{Lie}[a, b]$ have a unique extension to derivations of all of Lie $[a, b]$, and that in particular this is the case for the derivations in the image of $\mathfrak{g r t}$ and $\mathfrak{d} \mathfrak{s}$ (cf. section 2). This gives a direct interpretation of the two maps to derivations in the diagram (1.1.1) whose commutativity we prove.

The existence of the injection $\mathfrak{d} \mathfrak{s} \rightarrow \mathfrak{d} \mathfrak{s}_{\text {ell }}$ arose from an elliptic reinterpretation of a major theorem by Ecalle in mould theory. This reading of Ecalle's work and interpretation of some of his important results constitute one of the main goals of this paper in themselves. Indeed, it appears that Ecalle's seminal work in mould and multizeta theory has been largely ignored by the multiple zeta community ${ }^{3}$. This minimalist way of phrasing the main result shows that it could actually be stated and proved without even defining an elliptic double shuffle Lie algebra. However, this object is important in its own right, principally for the following reason. Recall that the usual double shuffle Lie algebra $\mathfrak{d s}$ expresses the double shuffle relations satisfied by the multiple zeta values, in the following sense. Let $\mathcal{F} \mathcal{Z}$, the formal multizeta algebra, be the graded dual of the universal enveloping algebra of $\mathfrak{d} \mathfrak{s}$; it is generated by formal symbols satisfying only the double shuffle relations. Since motivic and real multizeta values are known to satisfy them (see for example $[\mathrm{So}]), \mathcal{F Z}$ surjects onto the algebras of motivic and real multizeta values. These surjections are conjectured to be isomorphisms, i.e., it is conjectured that the double shuffle relations generate all algebraic relations between motivic, resp. real multizeta values (with the first of these problems being undoubtedly much more tractable than the second, for reasons of transcendence).

The role played by the double shuffle algebra with respect to ordinary multizeta values is analogous to the role played by the elliptic double shuffle algebra defined in this article with respect to the elliptic mzv's defined in [LMS]. There, we define an elliptic generating series in the completed Lie algebra Lie $[a, b]$, whose coefficients, called elliptic mzv's or emzv's, are related to the iterated integrals that form the coefficients of Enriquez' monodromic elliptic associator, and we give an explicit "dimorphic" or "double shuffle" type symmetry of this generating series which is exactly the defining property of $\mathfrak{d} \mathfrak{s}_{e l l}$. Indeed, letting $\mathcal{E}$ denote the graded Hopf algebra generated

3 According to the author's discussion with several colleagues, this appears to be at least partly due to a reluctance to accept Ecalle's language, because, at least according to some, it uses a system of words with varying vowels, rather than the more standard single letters, for the basic objects. This seems surprising, as it is unclear why calling a derivation arit(f), say, rather than $\mathrm{D}_{f}$ should pose such a problem. Possibly we enter here into the domain of psychology. A second, more serious obstacle is the lack of proofs in Ecalle's work, and the incredible profusion of statements, which makes it difficult to pick out exactly what is needed to establish a specific result. The author has attempted to solve this problem, at least partially, in the basic text [S] which gives an introduction with complete proofs to the portion of Ecalle's work most directly related to current problems in double shuffle algebra. 
by the emzv's, we show in [LMS] that the vector space $\mathfrak{n} \mathfrak{e}=\mathcal{E}_{>0} /\left(\mathcal{E}_{>0}\right)^{2}$ is isomorphic to a semidirect product $\mathfrak{b}_{3} \rtimes \mathfrak{n} \mathfrak{z}$ ", where $\mathfrak{n} \mathfrak{z}$ is the space of "new multizeta values" obtained by quotienting the algebra of multizeta values by $\zeta(2)$ and products. Under the standard conjecture from multizeta theory $\mathfrak{n z}^{\vee} \simeq \mathfrak{g r t}$, as well as Enriquez' conjecture $\mathfrak{r}_{\text {ell }} \simeq \mathfrak{b}_{3}$, this implies that $\mathfrak{n} \mathfrak{e} \simeq \mathfrak{g r t}_{\text {ell }}$. If $\mathfrak{g r t}_{\text {ell }} \simeq \mathfrak{d}_{\mathfrak{s}_{\text {ell }}}$, as we believe, this would mean that the elliptic double shuffle property determines all algebraic relations between the emzv's. This topic, which reflects the geometric aspects of the elliptic double shuffle relations introduced in this paper, is explored in detail in [LMS].

The content of the present paper has some relation with the recent preprint [Br3] as well as the earlier, closely related online lecture notes [Br2]. In particular Brown gives the existence of rational-function moulds satisfying the double shuffle relations, which is an immediate consequence of an important theorem of Ecalle that appears in all of his articles concerning ARI/GARI and multiple zeta values (cf. Theorem 1.3.2 below), although Brown introduces a completely different construction (vines and grapes). Brown also mentions in passing (cf. (3.7) of [Br3]) the result of the useful extension Lemma 2.1.2 below, however without proof. In [Br2] (conjecture 3) and [Br3] (following Prop. 4.6), Brown asks the question of whether $\mathfrak{u}^{\text {geom }} \simeq \mathfrak{p l s}$. The answer to this question is no; indeed all elements of $\mathfrak{g r t}$ with no depth 1 part furnish elements of $\mathfrak{p l s}$ not lying in $\mathfrak{u}$ via Enriquez' section, as explained in the Corollary following Theorem 1.3.3.

Acknowledgements. The work on this paper benefited from discussions with B. Enriquez and P. Lochak, both of whom listened patiently and provided some crucial elements of proof. J. Ecalle repeatedly gave of his time to help understand some of his results. R. Hain also shed some light on details arising from his motivic work. I thank them all warmly.

\subsection{The elliptic Grothendieck-Teichmüller Lie algebra}

In this section we recall the definition of the elliptic Grothendieck-Teichmüller Lie algebra $\mathfrak{g r t}_{\text {ell }}$ defined in [En1], along with some of its main properties. Recall that the genus 1 braid Lie algebra on $n$ strands, $\mathfrak{t}_{1, n}$, is generated by elements $x_{1}^{+}, \ldots, x_{n}^{+}$and $x_{1}^{-}, \ldots, x_{n}^{-}$subject to relations

$$
\begin{gathered}
x_{1}^{+}+\cdots+x_{n}^{+}=x_{1}^{-}+\cdots+x_{n}^{-}=0, \quad\left[x_{i}^{+}, x_{j}^{+}\right]=\left[x_{i}^{-}, x_{j}^{-}\right]=0 \text { if } \mathrm{i} \neq \mathrm{j}, \\
{\left[x_{i}^{+}, x_{j}^{-}\right]=\left[x_{j}^{+}, x_{i}^{-}\right] \text {for } i \neq j, \quad\left[x_{i}^{+},\left[x_{j}^{+}, x_{k}^{-}\right]\right]=\left[x_{i}^{-},\left[x_{j}^{+}, x_{k}^{-}\right]\right]=0 \text { for } i, j, k \text { distinct. }}
\end{gathered}
$$

We write $t_{i j}=\left[x_{i}^{+}, x_{j}^{-}\right]$. It is the Lie algebra of the unipotent completion of the topological fundamental group of the configuration space of $n$ ordered marked points on the torus (cf. [CEE, $\S 2.2$ ] for details). The Lie algebra $\mathfrak{t}_{1,2}$ is isomorphic to Lie $[a, b]$, the free Lie algebra on two generators ${ }^{4}$ $a$ and $b$. Throughout this article, we write $\operatorname{Lie}[a, b]$ for the completed Lie algebra, i.e., it contains infinite Lie series and not just polynomials. Thus an element $\alpha \in \mathfrak{t}_{1,2} \simeq \operatorname{Lie}[a, b]$ is a Lie series $\alpha(a, b)$ in two free variables.

Definition. The elliptic Grothendieck-Teichmüller Lie algebra $\mathfrak{g r t}_{\text {ell }}$ is the set of triples $\left(\psi, \alpha_{+}, \alpha_{-}\right)$ with $\psi \in \mathfrak{g r t}, \alpha_{+}, \alpha_{-} \in \mathfrak{t}_{1,2}$, such that setting

$$
\left\{\begin{array}{l}
\Psi\left(x_{1}^{ \pm}\right)=\alpha_{ \pm}\left(x_{1}^{ \pm}, x_{1}^{\mp}\right)+\left[x_{1}^{ \pm}, \psi\left(t_{12}, t_{23}\right)\right], \\
\Psi\left(x_{2}^{ \pm}\right)=\alpha_{ \pm}\left(x_{2}^{ \pm}, x_{2}^{\mp}\right)+\left[x_{2}^{ \pm}, \psi\left(t_{12}, t_{13}\right)\right], \\
\Psi\left(x_{3}^{ \pm}\right)=\alpha_{ \pm}\left(x_{3}^{ \pm}, x_{3}^{\mp}\right)
\end{array}\right.
$$

\footnotetext{
4 With respect to the notation of [En1] we have $\operatorname{Lie}[a, b]=\mathfrak{t}_{1,2}, a=y_{1}=x_{1}^{-}, b=x_{1}=x_{1}^{+}$(Enriquez uses both notations).
} 
yields a derivation of $\mathfrak{t}_{1,3}$. The space $\mathfrak{g r t}_{\text {ell }}$ is made into a Lie algebra by bracketing derivations; in other words, writing $D_{\alpha_{ \pm}}$for the derivation of $\mathfrak{t}_{1,2} \simeq \operatorname{Lie}[a, b]$ which takes $a \mapsto \alpha_{+}(a, b)$ and $b \mapsto \alpha_{-}(a, b)$, we have

$$
\left\langle\left(\psi, \alpha_{+}, \alpha_{-}\right),\left(\phi, \beta_{+}, \beta_{-}\right)\right\rangle=\left(\{\psi, \phi\}, D_{\alpha_{ \pm}}\left(\beta_{+}\right)-D_{\beta_{ \pm}}\left(\alpha_{+}\right), D_{\alpha_{ \pm}}\left(\beta_{-}\right)-D_{\beta_{ \pm}}\left(\alpha_{-}\right)\right),
$$

where $\{\psi, \phi\}$ is the Poisson (or Ihara) bracket on $\mathfrak{g r t}$. Finally, we assume that the coefficient of $a$ in both $\alpha_{+}$and $\alpha_{-}$is equal to 0 .

Remark. The last assumption is not contained in Enriquez' original definition. In particular he allows the element $(0,0, a)$, corresponding to the derivation $e(a)=0, e(b)=a$, which together with $\epsilon_{0}(a)=b, \epsilon_{0}(b)=0$ generate a copy of $\mathfrak{s l}_{2}$ in $\mathfrak{g r t}_{\text {ell }}$. Because of this, Enriquez' version of $\mathfrak{g r t}_{\text {ell }}$ is not pronilpotent, and is thus strictly larger than the Lie $\pi_{1}(M E M)$ studied in [HM], which is the Lie algebra of the prounipotent radical of the fundamental group of $M E M$. Thus, isomorphism can only be conjectured if the extra element is removed, motivating our slight alteration of his definition. We nonetheless write $\mathfrak{g r t}_{\text {ell }}$ for the modified version; the results of Enriquez on elements of $\mathfrak{g r t}_{\text {ell }}$ that we cite adapt directly with no changes.

We summarize Enriquez' important results concerning $\mathfrak{g r t}_{e l l}$ in the following theorem.

Theorem 1.2.1. (cf. [En1]) For all $\left(\psi, \alpha_{+}, \alpha_{-}\right) \in \mathfrak{g r t}_{\text {ell }}$, the derivation $D_{\alpha_{ \pm}}$of $\mathfrak{t}_{1,2}$ annihilates the element $t_{12}=[a, b]$. But for each $\psi \in \mathfrak{g r t}$, there exists one and only one triple $\left(\psi, \alpha_{+}, \alpha_{-}\right) \in \mathfrak{g r t}_{\text {ell }}$ such that $D_{\alpha_{ \pm}}$restricts to the Lie subalgebra Lie $\left[t_{01}, t_{12}\right]$ as follows:

$$
\left\{\begin{aligned}
t_{01} & \mapsto\left[\psi\left(t_{01}, t_{12}\right), t_{01}\right], \\
t_{02} & \mapsto\left[\psi\left(t_{02}, t_{12}\right), t_{02}\right], \\
t_{12} & \mapsto 0 .
\end{aligned}\right.
$$

The map $\gamma: \mathfrak{g r t} \rightarrow \mathfrak{g r t}_{\text {ell }}$ mapping $\psi$ to this triple is a Lie algebra morphism that is a section of the canonical surjection $\mathfrak{g r t}_{\text {ell }} \rightarrow \mathfrak{g r t}$. The Lie algebra $\mathfrak{g r t}_{\text {ell }}$ thus has a semi-direct product structure

$$
\mathfrak{g r t}_{\text {ell }}=\mathfrak{r}_{\text {ell }} \rtimes \gamma(\mathfrak{g r t}) .
$$

These results of Enriquez show that $\mathfrak{g r t}_{\text {ell }}$ is generated by elements belonging to two particular subspaces. The first is the subspace $\mathfrak{r}_{\text {ell }}$ of triples $\left(\psi, \alpha_{+}, \alpha_{-}\right)$with $\psi=0$, which forms a Lie ideal inside $\mathfrak{g r t}_{\text {ell }}$. The quotient $\mathfrak{g r t}$ ell $/ \mathfrak{r}_{\text {ell }}$ is canonically isomorphic to $\mathfrak{g r t}$, the surjection being nothing other than the morphism forgetting $\alpha_{+}$and $\alpha_{-}$. The second subspace, the image of the section $\mathfrak{g r t} \hookrightarrow \mathfrak{g r t}_{\text {ell }}$, is the space of triples that restrict on the free Lie subalgebra Lie $\left[t_{01}, t_{02}\right]$ to Ihara-type derivations (1.2.2). For any triple $\left(\psi, \alpha_{+}, \alpha_{-}\right)$of the second type, i.e., in the - but only and uniquely for those, not for general elements of $\mathfrak{g r t}_{e l l}-$ we let $D_{\psi}=D_{\alpha_{ \pm}}$, and write $\tilde{D}_{\psi}$ for the the restriction of $D_{\psi}$ to Lie $\left[t_{02}, t_{12}\right]$ given by (1.2.2).

Remark. This is actually a rephrasing of part of Enriquez' results. In fact, he gives the derivation $D_{\psi}$ by explicitly displaying its value on $t_{01}$ (as in (1.2.2) and on $b$. Since $D_{\psi}\left(t_{12}\right)=0$, the restriction of $D_{\psi}$ to Lie $\left[t_{01}, t_{02}\right]$ is the well-known Ihara derivation associated to $\psi \in \mathfrak{g r t}$, and therefore the value on $t_{02}$ must be as in (1.2.2). The fact that $D_{\psi}$ is the only extension of (1.2.2) to a derivation on all of Lie $[a, b]$ follows from our extension Lemma 2.1.2 below. This characterization of $D_{\psi}$ is sufficient for our purposes in this article; we do not actually use the explicit expression of $D_{\psi}(b)$, but it is necessary for Enriquez' work on elliptic associators.

The map

$$
\begin{aligned}
\mathfrak{g r t}_{\text {ell }} & \rightarrow \operatorname{Der}^{0} \operatorname{Lie}[a, b] \\
\left(\psi, \alpha_{+}, \alpha_{-}\right) & \mapsto D_{\alpha_{ \pm}}
\end{aligned}
$$


is injective; in other words, knowing the pair $\left(\alpha_{+}, \alpha_{-}\right)$allows us to uniquely recover $\psi$. This result follows from [T2, Theorem 1.17] (building on previous work in [NTU]), which states that removing the third braid strand yields an injection $\mathcal{D}_{1}^{(2)} \hookrightarrow \mathcal{D}_{1}^{(1)}$, where $\mathcal{D}_{1}^{(1)} \simeq \operatorname{Der}{ }^{0} \operatorname{Lie}[a, b]$ and $\mathcal{D}_{1}^{(2)}$ is a space of special derivations of $\mathcal{L}_{1}^{(2)} \simeq \mathfrak{t}_{1,3}$ which contains (and is conjecturally equal to) $\mathfrak{g r t}_{\text {ell }}$.

Furthermore, by Lemma 2.1.1 below, there is an injective linear map

$$
\begin{aligned}
\operatorname{Der}{ }^{0} \operatorname{Lie}[a, b] & \rightarrow \operatorname{Lie}[a, b] \\
D & \mapsto D(a),
\end{aligned}
$$

which is a Lie algebra bijection onto its image when that image (equal to the subspace Lie ${ }^{\text {push }}[a, b]$ of push-invariant elements of Lie $[a, b]$, cf. section 2) is equipped with the corresponding bracket. In particular this shows that in the triple $\left(\psi, \alpha_{+}, \alpha_{-}\right)$, the element $\alpha_{+}$determines $\alpha_{-}$, and thus also $\psi$. We write $\gamma_{+}: \mathfrak{g r t} \hookrightarrow \operatorname{Lie}[a, b]$ for the map sending $\psi \mapsto \alpha_{+}$. By the above arguments, $\gamma_{+}$ determines $\gamma$ and vice versa.

The desired triangle diagram (1.1.1) is thus equivalent to

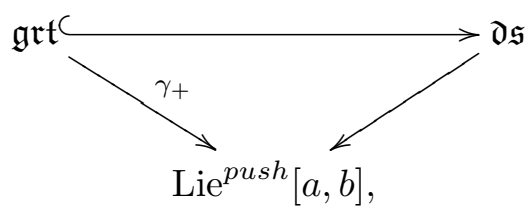

by composing it with the map (1.2.4). Our main result, Theorem 1.3.1 below, is the explicit version of the commutation of the diagram (1.2.5).

\subsection{Mould theory, elliptic double shuffle and the main theorem}

In this section we explain how we use Ecalle's mould theory - particularly adapted to the study of dimorphic (or "double shuffle") structures - to construct the elliptic double shuffle Lie algebra $\mathfrak{d} \mathfrak{s}_{e l l}$, which like $\mathfrak{g r t}_{\text {ell }}$ is a subspace of the push-invariant elements of $\operatorname{Lie}[a, b]$, and how we reinterpret one of Ecalle's major theorems and combine it with some results from Baumard's Ph.D. thesis ([B]), to define the injective Lie morphism $\mathfrak{d} \mathfrak{s} \rightarrow \mathfrak{d} \mathfrak{s}_{\text {ell }}$.

We assume some familiarity with moulds in this section; however all the necessary notation and definitions starting with that of a mould are recalled in the appendix at the end of the paper. We use the notation $A R I$ to denote the vector space of moulds with constant term 0 , and write $A R I_{l u}$ for $A R I$ equipped with the $l u$-bracket and $A R I_{\text {ari }}$ for $A R I$ equipped with the ari-bracket (the usual $A R I$ according to Ecalle's notation). Similarly, we write GARI for the set of moulds with constant term 1 and write $G A R I_{m u}$ and $G A R I_{g a r i}$ for the groups obtained by equipping GARI with the mu and gari multiplication laws. In section 3 we will introduce a third Lie bracket on $A R I$, the Dari-bracket, and employ the notation $A R I_{D a r i}$, as well as the corresponding group $G A R I_{\text {Dgari }}$ with multiplication law Dgari.

We define the following operators on moulds:

$$
\left\{\begin{array}{l}
\operatorname{dar}(P)\left(u_{1}, \ldots, u_{r}\right)=u_{1} \cdots u_{r} P\left(u_{1}, \ldots, u_{r}\right) \\
\operatorname{dur}(P)\left(u_{1}, \ldots, u_{r}\right)=\left(u_{1}+\cdots+u_{r}\right) P\left(u_{1}, \ldots, u_{r}\right) \\
\Delta(P)\left(u_{1}, \ldots, u_{r}\right)=u_{1} \cdots u_{r}\left(u_{1}+\cdots+u_{r}\right) P\left(u_{1}, \ldots, u_{r}\right) \\
\operatorname{ad}(Q) \cdot P=[Q, P] \text { for all } Q \in A R I .
\end{array}\right.
$$

We take $\operatorname{dar}(P)(\emptyset)=\operatorname{dur}(P)(\emptyset)=\Delta(P)(\emptyset)=P(\emptyset)$. The operators $d u r$ and ad $(Q)$ are derivations of the Lie algebra $A R I_{l u}$, whereas dar is an automorphism of $A R I_{l u}$. We will also 
make use of the inverse operators $d u r^{-1}$ (resp. $d u r^{-1}$ and $\Delta^{-1}$ ) defined by dividing a mould in depth $r$ by $\left(u_{1}+\cdots+u_{r}\right)$ (resp. by $\left(u_{1}+\cdots+u_{r}\right)$ and $\left.\left(u_{1}+\cdots+u_{r}\right) u_{1} \cdots u_{r}\right)$.

If $p \in \operatorname{Lie}[a, b]$, then we have

$$
\left\{\begin{array}{l}
m a([p, a])=d u r(m a(p)) \\
m a(p(a,[b, a]))=\operatorname{dar}(m a(p)) \\
m a([p(a,[b, a]), a])=\Delta(m a(p)) .
\end{array}\right.
$$

A proof of the first equality can be found in [R, Proposition 4.2.1.1] or [S, Lemma 3.3.1]. The second is obvious from the definition of $m a$ (cf. Appendix), since substituting $[b, a]$ for $b$ in $C_{k}$ yields $-C_{k+1}$ so making the substitution in a monomial $C_{k_{1}} \cdots C_{k_{r}}$ yields $(-1)^{r} C_{k_{1}+1} \cdots C_{k_{r}+1}$, and we have

$$
m a\left((-1)^{r} C_{k_{1}+1} \cdots C_{k_{r}+1}\right)=(-1)^{r}(-1)^{k_{1}+\cdots+k_{r}} u_{1}^{k_{1}} \cdots u_{r}^{k_{r}}=u_{1} \cdots u_{r} m a\left(C_{k_{1}} \cdots C_{k_{r}}\right) .
$$

The third equality of (1.3.2) follows from the first two.

We now recall the definition of the key mould pal that lies at the heart of much of Ecalle's theory of moulds. Following [E2], we start by introducing an auxiliary mould dupal $\in A R I$, given by the simple explicit expression

$$
\operatorname{dupal}\left(u_{1}, \ldots, u_{r}\right)=\frac{B_{r}}{r !} \frac{1}{u_{1} \cdots u_{r}}\left(\sum_{j=0}^{r-1}(-1)^{j}\left(\begin{array}{c}
r-1 \\
j
\end{array}\right) u_{j+1}\right)
$$

The mould $p a l$ is then defined by setting $\operatorname{pal}(\emptyset)=1$ and using the equality

$$
d u r(p a l)=p a l d u p a l,
$$

which gives a recursive definition for pal depth by depth starting with $\operatorname{pal}(\emptyset)=1$, since to determine the left-hand side $\operatorname{dur}(\mathrm{pal})$ in depth $r$ only requires knowing pal up to depth $r-1$ on the right-hand side.

Since $\operatorname{pal}(\emptyset)=1$, we have pal $\in G A R I$. We write invpal for its inverse $i n v_{\text {gari }}($ pal $)$ in the group $G A R I_{\text {gari }}$. Since $G A R I_{\text {gari }}$ is the exponential of the Lie algebra $A R I_{\text {ari }}$, it has an adjoint action on $A R I_{\text {ari }}$; we write $A d_{\text {ari }}(P)$ for the adjoint operator on $A R I_{\text {ari }}$ associated to a mould $P \in G A R I_{\text {gari }}$.

At this point we are already equipped to baldly state our main theorem linking Ecalle's theory of moulds to Enriquez' section $\gamma: \mathfrak{g r t} \rightarrow \mathfrak{g r t}_{e l l}$, or rather to the modified version $\gamma_{+}$introduced above that maps $\psi$ to the associated element $\alpha_{+}$in Enriquez' triple $\left(\psi, \alpha_{+}, \alpha_{-}\right)$.

Theorem 1.3.1. Let $\psi \in \mathfrak{g r t}$ and set $f(x, y)=\psi(x,-y)$. We have the following equality of moulds:

$$
\Delta\left(A d_{\text {ari }}(\text { invpal }) \cdot m a(f)\right)=m a\left(\gamma_{+}(\psi)\right) .
$$

In order to place this theorem in context and explain its power in terms of helping to define an elliptic double shuffle Lie algebra that in turn will shed light on the dimorphic ("double-shuffle") properties of elliptic multiple zeta values, we first give some results from the literature, starting with Ecalle's main theorem, with which he first revealed the surprising role of the adjoint operator $A d_{a r i}(p a l)$ and its inverse $A d_{a r i}(\text { pal })^{-1}=A d_{\text {ari }}($ invpal $)$. 
Recall from the appendix that in terms of moulds, $\mathfrak{d} \mathfrak{s}$ is isomorphic to the Lie subalgebra of $A R I_{\text {ari }}$ of polynomial-valued moulds that are even in depth 1 , and are alternal with swap that is alternil up to addition of a constant mould. The notation we use for this in mould language is a bit heavy, but has the advantage of concision and total precision in that the various symbols attached to $A R I$ carry all of the information about the moulds in the subspace under consideration: we have the isomorphism

$$
m a: \mathfrak{d} \mathfrak{s} \stackrel{m a}{\rightarrow} A R I_{\text {ari }}^{\text {pol } \underline{a l} * \underline{i l},},
$$

where pol indicates polynomial moulds, the underlining is Ecalle's notation for moulds that are even in depth 1 , and the usual notation $a l / i l$ for an alternal mould with alternil swap is weakened to $a l * i l$ when the swap is only alternil up to addition of a constant mould.

Similarly, the notation $A R I_{a r i}^{a l * a l}$ refers to the subspace of moulds in $A R I_{a r i}$ that are even in depth 1 and alternal with swap that is alternal up to addition of a constant mould (or "bialternal"). When we consider the subspace of these moulds that are also polynomial-valued, $A R I^{p o l, a l * a l}$, we obtain the (image under $m a$ of the) "linearized double shuffle" space $\mathfrak{l}$ s studied for example in [Br3]. But the full non-polynomial space is of course hugely larger. One of Ecalle's most remarkable discoveries is that the mould pal provides an isomorphism between the two types of dimorphy, as per the following theorem.

Theorem 1.3.2. (cf. $\left.[\mathrm{E}]^{5}\right)$ The adjoint map Adari (invpal) induces a Lie isomorphism of Lie subalgebras of $A R I_{\text {ari }}$ :

$$
A d_{\text {ari }}(\text { invpal }): A R I_{\text {ari }}^{a l} \stackrel{i l}{\rightarrow} \stackrel{\sim}{\rightarrow} A R I_{\text {ari }}^{a l} \stackrel{a l}{ },
$$

and if $F \in A R I \underline{a l} * \underline{i l}$ and $C$ is the constant mould such that $\operatorname{swap}(F+C)$ is alternil, then $\operatorname{swap}\left(A d_{\text {ari }}(\right.$ invpal $\left.)(F)\right)+C$ is alternal, i.e., the constant corrections for $F$ and $A d_{\text {ari }}($ invpal $) \cdot F$ are the same. In particular if $C=0$, i.e., if $F$ is $\underline{\text { al }} / \underline{i l}$, then $A d_{\text {ari }}($ invpal $)(F)$ lies in $\underline{\text { al }} / \underline{a l}$.

One important point to note in the result of Theorem 1.3.2 is that the operator $A d_{\text {ari }}($ invpal $)$ does not respect polynomiality of moulds. Indeed, applying $A d_{\text {ari }}(p a l)$ to bialternal polynomial moulds produces quite complicated denominators with many factors. However, in his doctoral thesis S. Baumard was able to show that conversely, when applying $A d_{\text {ari }}($ invpal $)$ to moulds $m a(f)$

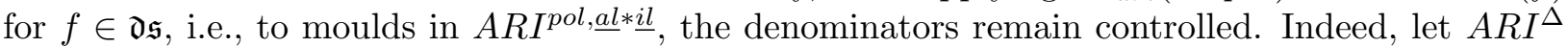
denote the space of moulds $P \in A R I$ such that $\Delta(P) \in A R I^{\text {pol }}$, i.e., the space of rational-function valued moulds whose denominator is "at worst" $u_{1} \cdots u_{r}\left(u_{1}+\cdots+u_{r}\right)$ in depth $r$.

Theorem 1.3.3. [B, Thms. 3.3, 4.35] The space ARI ${ }^{\Delta}$ forms a Lie algebra under the ari-bracket, and we have an injective Lie algebra morphism

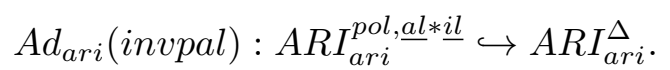

Recall that $\mathfrak{p l s}$ ("polar linearized double shuffle") is the notation used by F. Brown for the space $A R I^{\Delta, \underline{a l} / \underline{a l}}$ and $\mathfrak{u}$ for the Lie subalgebra of $A R I$ generated by $B_{-2}$ and $B_{2 i}$ for $i \geq 1$, where $B_{i}$ denotes the mould concentrated in depth 1 defined by $B_{i}\left(u_{1}\right)=u_{1}^{i}$. As a corollary of Theorems 1.3.1, 1.3.2 and 1.3.3, we give a negative answer to the question posed by Brown ([Br2], conjecture 3 and [Br3], following Prop. 4.6) as to whether $\mathfrak{p l s}$ and $\mathfrak{u}$ are equal.

\footnotetext{
5 This result is stated and used constantly in [E], as well as many other analogous results concerning other symmetries. But the proof is not given. Ecalle was kind enough to send us a sketch of the proof in a personal letter, relying on the fundamental identity (2.62) of [E], itself not proven there. Full details of the reconstructed proof can be found in [S], with (2.62) proved in Theorem 2.8.1 and Theorem 1.3.2 above proved in $\S 4.6$.
} 
Corollary. Let $\psi \in \mathfrak{g r t}$ be an element of $\mathfrak{g r t}$ having no depth 1 part. Then

$$
\Delta^{-1}\left(m a\left(\gamma_{+}(\psi)\right) \in A R I^{\Delta, \underline{a l} / \underline{a l}}=\mathfrak{p l s}\right.
$$

but

$$
\Delta^{-1}\left(\operatorname{ma}\left(\gamma_{+}(\psi)\right)\right) \notin \mathfrak{u} .
$$

Proof. Since by Furusho's theorem, $\psi(x, y) \mapsto f(x, y)=\psi(x,-y)$ maps $\mathfrak{g r t} \hookrightarrow \mathfrak{d s}$, we have

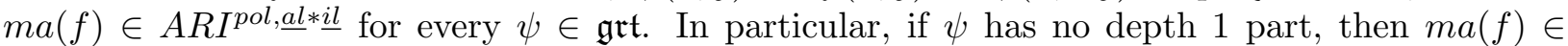

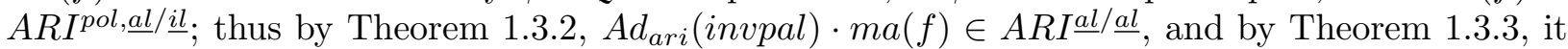

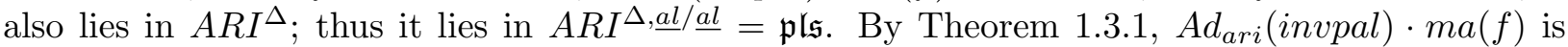
equal to $\Delta^{-1}\left(m a\left(\gamma_{+}(\psi)\right)\right.$ where $\gamma_{+}$denotes Enriquez' section $\mathfrak{g r t} \rightarrow \mathfrak{g r t}_{\text {ell }}$, associating to $\psi \in \mathfrak{g r t}$ the element $\alpha_{+}$from the triple $\left(\psi, \alpha_{+}, \alpha_{-}\right)$. But Enriquez shows that $\mathfrak{g r t}_{\text {ell }}$ is a semi-direct product $\gamma_{+}(\mathfrak{g r t}) \rtimes \mathfrak{r}_{e l l}$ and that $\Delta(\mathfrak{u}) \subset m a\left(\mathfrak{r}_{e l l}\right)$. Thus $m a\left(\gamma_{+}(\mathfrak{g r t})\right) \cap \Delta(\mathfrak{u})=\{0\}$.

For the rest of this article we will use the notation:

$$
\left\{\begin{array}{l}
f=\psi(x,-y) \\
F=m a(f) \\
A=A d_{\text {ari }}(\text { invpal }) \cdot F \\
M=\Delta(A) .
\end{array}\right.
$$

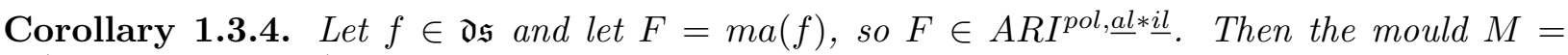
$\Delta\left(A d_{\text {ari }}(\right.$ invpal $\left.) \cdot F\right)$ is alternal, push-invariant and polynomial-valued.

Proof. Let $A=A d_{\text {ari }}($ invpal) $\cdot F$. Then $A \in A R I \underline{a l} * \underline{a l}$ by Theorem 1.3.2, so $A$ is alternal, and furthermore $A$ is push-invariant because all moulds in $A R I \underline{a l} * \underline{a l}$ are push-invariant (see [E2] or [S, Lemma 2.5.5]). Thus $M=\Delta(A)$ is also alternal and push-invariant since $\Delta$ preserves these properties. The fact that $M$ is polynomial-valued follows from Theorem 1.3.3.

Definition. A mould $P$ is said to be $\Delta$-bialternal if $\Delta^{-1}(P)$ is bialternal, i.e., $P \in \Delta\left(A R I_{\text {ari }}^{a l * a l}\right)$. The elliptic double shuffle Lie algebra $\mathfrak{d} \mathfrak{s}_{\text {ell }} \subset \operatorname{Lie}[a, b]$ is the set of Lie polynomials which map under $m a$ to polynomial-valued $\Delta$-bialternal moulds that are even in depth 1, i.e.,

$$
\mathfrak{d} \mathfrak{s}_{e l l}=m a^{-1}\left(\Delta\left(A R I_{a r i}^{\Delta, \underline{a l} * \underline{a l}}\right)\right) .
$$

Taken together, Theorems 1.3.2 and 1.3.3 show that the image of $m a(\mathfrak{d s})=A R I_{\text {ari }}^{\text {pol, } \underline{a l} * \underline{i l}}$ under $A d_{\text {ari }}\left(\right.$ invpal) lies in $A R I_{\text {ar } \underline{\Delta} \underline{a} * \underline{a l}}$, so the image under $\Delta \circ A d_{\text {ari }}($ invpal) lies in the space of polynomial-valued $\Delta$-bialternal moulds that are also even in depth 1 (since it is easy to see that $A d_{a r i}\left(\right.$ invpal) preserves the lowest-depth part of a mould). Thus we can define $\gamma_{s}$ to be the polynomial avatar of $\Delta \circ A d_{\text {ari }}($ invpal $)$, i.e., $\gamma_{s}$ is defined by the commutation of the diagram

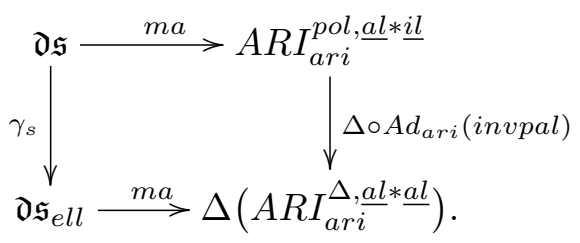

Thus for $f \in \mathfrak{d} \mathfrak{s}$ we have

$$
m a\left(\gamma_{s}(f)\right)=\Delta\left(\text { Ad }_{\text {ari }}(\text { invpal }) \cdot m a(f)\right) .
$$


This reduces the statement of the main Theorem 1.3.1 above to the equality

$$
\gamma_{s}(f)=\gamma_{+}(\psi),
$$

i.e., to the commutation of the diagram

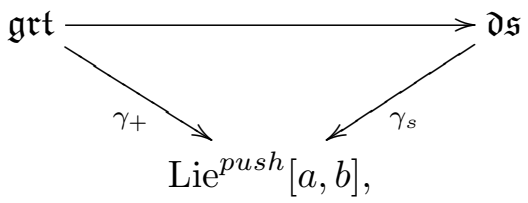

which is the precise version of the desired diagram (1.2.5).

As a final observation, we note that the definition of $\mathfrak{d} \mathfrak{s}_{\text {ell }}$ makes the injective Lie algebra morphism $\mathfrak{b}_{3} \hookrightarrow \mathfrak{d} \mathfrak{s}_{\text {ell }}$ mentioned at the beginning of the introduction obvious. Indeed, identifying $\mathfrak{b}_{3}$ with its image in Lie ${ }^{\text {push }}[a, b]$ under the map (1.2.4), it is generated by the polynomials $\epsilon_{2 i}(a)=$ $a d(a)^{2 i}(b)=C_{2 i+1}$ for $i \geq 0$, which map under $m a$ to the moulds $B_{2 i}$ concentrated in depth 1 and given by $B_{2 i}\left(u_{1}\right)=u_{1}^{2 i}$ (Ecalle denotes these moulds by ekma $a_{2 i}$ at least for $i \geq 1$; note however that $B_{0}$ and $\Delta^{-1}\left(B_{0}\right)=B_{-2}$ are essential in the elliptic situation). To show that these moulds lie in $\mathfrak{d} \mathfrak{s}_{\text {ell }}$, we need only note that the moulds $\Delta^{-1}\left(B_{2 i}\right)=B_{2 i-2}$ are even in depth 1 , and trivially bialternal since this condition is empty in depth 1 .

\section{Proof of the main theorem}

For the proof of the main theorem, we first recall in 2.1 a few well-established facts about non-commutative polynomials, moulds and derivations, and give the key lemma about extending derivations on the Lie subalgebra $\operatorname{Lie}\left[t_{01}, t_{02}\right]$ to all of Lie $[a, b]$. Once these ingredients are in place, the proof of the main theorem, given in 2.2 , is a simple consequence of one important proposition, whose proof, contained in section 3, necessitates some developments in mould theory. In fact, the present section could be written entirely in terms of polynomials in $a$ and $b$ without any reference to moulds. We only use moulds in the proof of Lemma 2.1.1, but merely as a convenience, as even this result could be stated and proved in terms of polynomials. Indeed this has already been done (cf. [S2]), but the proof given here using moulds is actually more elegant and simple.

\subsection{The push-invariance and extension lemmas}

Definition. For $p \in \operatorname{Lie}[a, b]$, write $p=p_{a} a+p_{b} b$ and set

$$
p^{\prime}=\sum_{i \geq 0} \frac{(-1)^{i-1}}{i !} a^{i} b \partial_{a}^{i}\left(p_{a}\right)
$$

where $\partial_{a}(a)=1, \partial_{a}(b)=0$. We call $p^{\prime}$ the partner of $p$. If $P \in A R I$ then we define $P^{\prime}$ to be the mould partner of $P$, given by the formula

$$
P^{\prime}\left(u_{1}, \ldots, u_{r}\right)=\frac{1}{u_{1}+\cdots+u_{r}}\left(P\left(u_{2}, \ldots, u_{r-1},-u_{1}-\cdots-u_{r-1}\right)-P\left(u_{2}, \ldots, u_{r}\right)\right) .
$$

This formula defines a partner for any mould $P \in A R I$, but in the case of polynomial-valued moulds it corresponds to (2.1.1) in the sense that if $P=m a(p)$, then $P^{\prime}=m a\left(p^{\prime}\right)$.

Recall that the push-operator on a mould is an operator of order $r+1$ in depth $r$ defined by

$$
\operatorname{push}(P)\left(u_{1}, \ldots, u_{r}\right)=P\left(-u_{1}-\cdots-u_{r}, u_{1}, \ldots, u_{r-1}\right),
$$


and that a mould $P$ is said to be push-invariant if $P=\operatorname{push}(P)$. We say that a polynomial $p \in \operatorname{Lie}[a, b]$ is push-invariant if $m a(p)$ is.

Lemma 2.1.1. Let $p, p^{\prime}$ be two polynomials in Lie $[a, b]$ such that the coefficient of a in $p$ and $p^{\prime}$ is zero, and let $D$ denote the derivation of Lie $[a, b]$ given by $a \mapsto p, b \mapsto p^{\prime}$. Then $D([a, b])=0$ if and only if $p$ is push-invariant and $p^{\prime}$ is its partner.

Proof. Let $P=m a(p)=m a(D(a))$ and $P^{\prime}=m a\left(p^{\prime}\right)=m a(D(b))$. Using the fact that $m a$ is a Lie algebra morphism (see Appendix) and the first identity of (1.3.2) we find that

$$
m a\left(D([a, b])=m a([D(a), b]+[a, D(b)])=[P, B]-d u r\left(P^{\prime}\right),\right.
$$

where $B=m a(b)$ is the mould concentrated in depth 1 given by $B\left(u_{1}\right)=1$. Note that the mould $[P, B]-\operatorname{dur}\left(P^{\prime}\right)$ is zero in depths $r \leq 1$.

Let us first assume that $P$ is push-invariant and $P^{\prime}$ is its partner as given in (2.1.2). We have

$$
[P, B]\left(u_{1}, \ldots, u_{r}\right)=P\left(u_{1}, \ldots, u_{r-1}\right)-P\left(u_{2}, \ldots, u_{r}\right)
$$

and

$$
\operatorname{dur}\left(P^{\prime}\right)=P\left(u_{2}, \ldots, u_{r}\right)-P\left(u_{2}, \ldots, u_{r-1},-u_{1}-\cdots-u_{r-1}\right) .
$$

Thus $[P, B]-\operatorname{dur}\left(P^{\prime}\right)$ is given in depth $r>1$ by

$$
P\left(u_{1}, \ldots, u_{r-1}\right)-P\left(u_{2}, \ldots, u_{r-1},-u_{1}-\cdots-u_{r-1}\right)=\left(P-p u s h^{-1}(P)\right)\left(u_{1}, \ldots, u_{r}\right),
$$

but since $P$ is push-invariant, this is equal to zero, so by $(2.1 .3) D([a, b])=0$.

Assume now that $D([a, b])=0$, i.e., $[P, B]=\operatorname{dur}\left(P^{\prime}\right)$, i.e.,

$$
P\left(u_{1}, \ldots, u_{r-1}\right)-P\left(u_{2}, \ldots, u_{r}\right)=\left(u_{1}+\cdots+u_{r}\right) P^{\prime}\left(u_{1}, \ldots, u_{r}\right) .
$$

This actually functions as a defining equation for $P^{\prime}$. But knowing that $P^{\prime}=m a\left(p^{\prime}\right)$ is a polynomial-valued mould, $(2.1 .6)$ implies that $P\left(u_{1}, \ldots, u_{r-1}\right)-P\left(u_{2}, \ldots, u_{r}\right)$ must vanish along the pole $u_{1}+\cdots+u_{r}=0$, in other words when $u_{r}=-u_{1}-\cdots-u_{r-1}$, so we have

$$
P\left(u_{1}, \ldots, u_{r-1}\right)=P\left(u_{2}, \ldots, u_{r-1},-u_{1}-\cdots-u_{r-1}\right) .
$$

As noted above, the right-hand side of (2.1.7) is nothing other than $p_{u s h}^{-1}(P)$, so $(2.1 .7)$ shows that $P$ is push-invariant. Furthermore, we can substitute (2.1.7) into the left-hand side of (2.1.6) to find the new defining equation for $P^{\prime}$ :

$$
P^{\prime}\left(u_{1}, \ldots, u_{r}\right)=\frac{1}{u_{1}+\cdots+u_{r}}\left(P\left(u_{2}, \ldots, u_{r-1},-u_{1}-\cdots-u_{r-1}\right)-P\left(u_{2}, \ldots, u_{r}\right)\right),
$$

but this coincides with (2.1.2), showing that $P^{\prime}$ is the partner of $P$.

Lemma 2.1.2 Let $\tilde{D}$ be a derivation of the Lie subalgebra $\operatorname{Lie}\left[t_{01}, t_{02}\right] \subset \operatorname{Lie}[a, b]$. Then

(i) there exists a unique derivation $D \in \operatorname{Der}^{0} \operatorname{Lie}[a, b]$ having the following two properties:

(i.1) $D\left(t_{02}\right)=\tilde{D}\left(t_{02}\right)$;

(i.2) $D(b)$ is the partner of $D(a)$.

(ii) If $\tilde{D}\left(t_{12}\right)=0$ and $D(a)$ is push-invariant, then $D$ is the unique extension of $\tilde{D}$ to all of Lie $[a, b]$. 
Proof. (i) Let $T=\tilde{D}\left(t_{02}\right)$, and write $T=\sum_{n \geq w} T_{n}$ for its homogeneous parts of weight $n$, where the weight is the degree as a polynomial in $a$ and $b$, and $w$ is the minimal weight occurring in $T$. We will construct a derivation $D$ satisfying $D\left(t_{02}\right)=\tilde{D}\left(t_{02}\right)$ via the equality

$$
\begin{aligned}
T= & D\left(\operatorname{Ber}_{-b}(a)\right) \\
= & D\left(a+\frac{1}{2}[b, a]+\frac{1}{12}[b,[b, a]]-\frac{1}{720}[b,[b,[b,[b, a]]]]+\cdots\right) \\
= & D(a)+\frac{1}{12}[D(b),[b, a]]-\frac{1}{720}\left[D(b),[b,[b,[b, a]]]-\frac{1}{720}[b,[D(b),[b,[b, a]]]]\right. \\
& \quad-\frac{1}{720}[b,[b,[D(b),[b, a]]]]+\cdots
\end{aligned}
$$

We construct $D(a)$ by solving (2.1.9) in successive weights starting with $w$. We start by setting $D(a)_{w}=T_{w}$ and $D(a)_{w+1}=T_{w+1}$, and take $D(b)_{w}$ and $D(b)_{w+1}$ to be their partners. We then continue to solve the successive weight parts of (2.1.9) for $D(a)$ in terms of $T$ and lower weight parts of $D(b)$. For instance the next few steps after weights $w$ and $w+1$ are given by

$$
\begin{aligned}
D(a)_{w+2}=T_{w+2} & -\frac{1}{12}\left[D(b)_{w},[b, a]\right] \\
D(a)_{w+3}=T_{w+3} & -\frac{1}{12}\left[D(b)_{w+1},[b, a]\right] \\
D(a)_{w+4}=T_{w+4} & -\frac{1}{12}\left[D(b)_{w+2},[b, a]\right]+\frac{1}{720}\left[D(b)_{w},[b,[b,[b, a]]]\right. \\
& +\frac{1}{720}\left[b,\left[D(b)_{w},[b,[b, a]]\right]\right]+\frac{1}{720}\left[b,\left[b,\left[D(b)_{w},[b, a]\right]\right]\right] .
\end{aligned}
$$

In this way we construct the unique Lie series $D(a)$ and its partner $D(b)$ such that the derivation $D$ satisfies $D\left(\operatorname{Ber}_{-b}(a)\right)=D\left(t_{02}\right)=T=\tilde{D}\left(t_{02}\right)$. We note that $D$ is not necessarily an extension of $\tilde{D}$ to all of Lie $[a, b]$, because $D$ and $\tilde{D}$ may not agree on $t_{12}$.

For (ii), suppose that $\tilde{D}\left(t_{12}\right)=\tilde{D}([a, b])=0$. Since $D(a)$ is push-invariant and $D(a)$ and $D(b)$ are partners by construction, we also have $D([a, b])=0$ by Lemma 2.1.1. Therefore $D$ and $\tilde{D}$ agree on $t_{02}$ and $t_{12}$, so on all of Lie $\left[t_{02}, t_{12}\right]$; thus $D$ is an extension of $\tilde{D}$. For the uniqueness, suppose that $E$ is another derivation of Lie $[a, b]$ that coincides with $\tilde{D}$ on $t_{02}$ and $t_{12}$. The fact that $E\left(t_{12}\right)=E([a, b])=0$ shows that $E(a)$ and $E(b)$ are partners by Lemma 2.1.1. But then $E$ satisfies (i.1) and (i.2), so it coincides with $D$.

\subsection{Proof of the main theorem.}

For each $\psi \in \mathfrak{g r t}$, let $f(x, y)=\psi(x,-y)$. Let $A=A d_{\text {ari }}($ invpal $) \cdot m a(f)$ as before, and $M=\Delta(A)$. By Corollary 1.3.4, there exists a polynomial $m \in \operatorname{Lie}[C]$ such that

$$
m a(m)=M=\Delta\left(A d_{\text {ari }}(\text { invpal }) \cdot m a(f)\right) .
$$

Since by the same corollary $m$ is push-invariant, we see that by Lemma 2.1.1 there exists a unique derivation $E_{\psi} \in \operatorname{Der} \operatorname{Lie}[a, b]$ such that $E_{\psi}(a)=m, E_{\psi}([a, b])=0$ and $E_{\psi}(b) \in \operatorname{Lie}[C]$, namely the one such that $E_{\psi}(b)$ is the partner of $E_{\psi}(a)$. The main result we need about this derivation is the following.

Proposition 2.2.1. The derivation $E_{\psi}$ satisfies

$$
E_{\psi}\left(t_{02}\right)=\left[\psi\left(t_{02}, t_{12}\right), t_{02}\right]
$$


Using this, we can easily prove the main theorem. Since $t_{12}=[a, b]$, we have $E_{\psi}\left(t_{12}\right)=0$, so Proposition 2.2.1 shows that $E_{\psi}$ restricts to a derivation $\tilde{E}_{\psi}$ on the Lie subalgebra Lie $\left[t_{02}, t_{12}\right]$, where it coincides with the restriction $\tilde{D}_{\psi}$ of Enriquez' derivation $D_{\psi}$ given in (1.2.2). Furthermore, since $E_{\psi}\left(t_{12}\right)=0$ and $E_{\psi}(a)=m$ is push-invariant, we are in the situation of Lemma 2.1.2 (ii), so $E_{\psi}$ is the unique extension of $\tilde{E}_{\psi}$ to all of $\operatorname{Lie}[a, b]$. But Enriquez' derivation $D_{\psi}$ is an extension of $\tilde{D}_{\psi}$ to all of Lie $[a, b]$, and it also satisfies $D_{\psi}\left(t_{12}\right)=0$, so by Lemma 2.1.1, $D_{\psi}(a)=\alpha_{+}=\gamma_{+}(\psi)$ is push-invariant; thus by Lemma 2.1.2 (ii) $D_{\psi}$ is the unique extension of $\tilde{D}_{\psi}$ to all of Lie $[a, b]$. Thus, since $\tilde{E}_{\psi}=\tilde{D}_{\psi}$, we must have $E_{\psi}=D_{\psi}$, and in particular $E_{\psi}(a)=m=D_{\psi}(a)=\gamma_{+}(\psi)$. Taking $m a$ of both sides yields the desired equality (1.3.5).

\section{Proof of Proposition 2.2.1}

\subsection{Mould theoretic derivations}

We begin by defining a mould-theoretic derivation $\mathcal{E}_{\psi}$ on $A R I_{l u}$ for each $\psi \in \mathfrak{g r t}$ as follows.

Definition. For any mould $P$, let $\operatorname{Darit}(P)$ be the operator on moulds defined by

$$
\operatorname{Darit}(P)=-\operatorname{dar}\left(\operatorname{arit}\left(\Delta^{-1}(P)\right)-\operatorname{ad}\left(\Delta^{-1}(P)\right)\right) \circ \operatorname{dar}^{-1} .
$$

Then for all $P, \operatorname{Darit}(P)$ is a derivation of $A R I_{l u}$, since $\operatorname{arit}(P)$ and $\operatorname{ad}(P)$ are both derivations and $d a r$ is an automorphism.

Let $\psi \in \mathfrak{g r t}$. We use the notation of (1.3.8), and set

$$
\mathcal{E}_{\psi}=\operatorname{Darit}(M)
$$

Recall that $A R I$ denotes the vector space of rational-valued moulds with constant term 0 . Let $A R I^{a}$ denote the vector space obtained by adding a single generator $a$ to the vector space $A R I$, and let $A R I_{l u}^{a}$ be the Lie algebra formed by extending the $l u$-bracket to $A R I^{a}$ via the relation

$$
[Q, a]=\operatorname{dur}(Q)
$$

for every $Q \in A R I_{l u}$. Recall from (1.3.2) that this equality holds in the polynomial sense if $Q$ is a polynomial-valued mould; in other words, (1.3.3) extends to an injective Lie algebra morphism $m a: \operatorname{Lie}[a, b] \rightarrow A R I_{l u}^{a}$ by formally setting $m a(a)=a$.

The Lie algebra $A R I_{l u}$ forms a Lie ideal of $A R I_{l u}^{a}$, i.e., there is an exact sequence of Lie algebras

$$
0 \rightarrow A R I_{l u} \rightarrow A R I_{l u}^{a} \rightarrow \mathbb{Q} a \rightarrow 0 .
$$

We say that a derivation (resp. automorphism) of $A R I_{l u}$ extends to a if there is a derivation (resp. automorphism) of $A R I_{l u}^{a}$ that restricts to the given one on the Lie subalgebra $A R I_{l u}$. To check whether a given derivation (resp. automorphism) extends to $a$, it suffices to check that relation (3.1.3) is respected.

Recall that $B=m a(b)$ is the mould concentrated in depth 1 given by $B\left(u_{1}\right)=1$. Let us write $B_{i}, i \geq 0$, for the mould concentrated in depth 1 given by $B_{i}\left(u_{1}\right)=u_{1}^{i}$. In particular $B_{0}=B=m a(b)$, and $B_{1}\left(u_{1}\right)=u_{1}$, so $B_{1}=m a([b, a])$.

Lemma 3.1.1. (i) The automorphism dar extends to a taking the value dar $(a)=a$;

(ii) The derivation dur extends to a taking the value dur $(a)=0$; 
(iii) For all $P \in A R I$, the derivation arit $(P)$ of $A R I_{l u}$ extends to $a$, taking the value arit $(P) \cdot a=0$.

(iv) For all $P \in A R I$, the derivation Darit $(P)$ of $A R I_{l u}$ extends to a, with $\operatorname{Darit}(P) \cdot a=P$. Furthermore, Darit $(P) \cdot B_{1}=0$.

Proof. Since dar is an automorphism, to check (3.1.3) we write

$$
[\operatorname{dar}(Q), \operatorname{dar}(a)]=[\operatorname{dar}(Q), a]=\operatorname{dur}(\operatorname{dar}(Q)) .
$$

But it is obvious from their definitions that dur and dar commute, so this is indeed equal to $\operatorname{dar}(\operatorname{dur}(Q))$. This proves (i). We check (3.1.3) for (ii) similarly. Because $\operatorname{dur}(a)=0$ and $d u r$ is a derivation, we have

$$
\operatorname{dur}([Q, a])=[\operatorname{dur}(Q), a]=\operatorname{dur}(\operatorname{dur}(Q))
$$

For (iii), we have

$$
\operatorname{arit}(P) \cdot[Q, a]=[\operatorname{arit}(P) \cdot Q, a]=\operatorname{dur}(\operatorname{arit}(P) \cdot Q)) .
$$

But as pointed out by Ecalle [E2] (cf. [S, Lemma 4.2.2] for details), arit $(P)$ commutes with dur for all $P$, which proves the result.

For (iv), the calculation to check that (3.1.3) is respected is a little more complicated. Let $Q \in A R I$. Again using the commutation of $\operatorname{arit}(P)$ with $d u r$, as well as that of dar and dur, we compute

$$
\begin{aligned}
& \operatorname{Darit}(P) \cdot[Q, a]=[\operatorname{Darit}(P)(Q), a]+[Q, \operatorname{Darit}(P)(a)] \\
& =\operatorname{dur}(\operatorname{Darit}(P) \cdot Q)+[Q, P] \\
& =-\operatorname{dur}\left(\operatorname{dar}\left(\operatorname{arit}\left(\Delta^{-1}(P)\right) \cdot \operatorname{dar}^{-1}(Q)-\left[\Delta^{-1}(P), \operatorname{dar}^{-1}(Q)\right)\right]\right)+[Q, P] \\
& =-\operatorname{dur}\left(\operatorname{dar}\left(\operatorname{arit}\left(\Delta^{-1}(P)\right) \cdot \operatorname{dar}^{-1}(Q)\right)\right)-\operatorname{dur}\left(\left[Q, \operatorname{dur}^{-1}(P)\right]\right)+[Q, P] \\
& =-\operatorname{dar}\left(\operatorname{dur}\left(\operatorname{arit}\left(\Delta^{-1}(P)\right) \cdot \operatorname{dar}^{-1}(Q)\right)\right)-[[Q, N], a]+[Q,[N, a]] \\
& \text { with } N=d u r^{-1} P \text {, i.e., } P=[N, a] \\
& =-\operatorname{dar}\left(\operatorname{arit}\left(\Delta^{-1}(P)\right) \cdot \operatorname{durdar} \operatorname{da}^{-1}(Q)\right)-[[Q, a], N] \text { by Jacobi } \\
& =-\operatorname{dar}\left(\operatorname{arit}\left(\Delta^{-1}(P)\right) \cdot \operatorname{dar}^{-1} \operatorname{dur}(Q)\right)-\left[\operatorname{dur}(Q), \operatorname{dur}^{-1} P\right] \\
& =-d a r\left(\operatorname{arit}\left(\Delta^{-1}(P)\right) \cdot d a r^{-1} \operatorname{dur}(Q)\right)-\operatorname{dar}\left(\left[\operatorname{dar}^{-1} \operatorname{dur}(Q), \operatorname{dar}^{-1} d u r^{-1}(P)\right]\right) \\
& =-\operatorname{dar}\left(\operatorname{arit}\left(\Delta^{-1}(P)\right) \cdot \operatorname{dar} r^{-1} \operatorname{dur}(Q)\right)+\operatorname{dar}\left(\left[\Delta^{-1}(P), \operatorname{dar}^{-1} d u r(Q)\right]\right) \\
& =\operatorname{Darit}(P) \cdot \operatorname{dur}(Q) \text {. }
\end{aligned}
$$

This proves the first statement of (iv). For the second statement, we note that $d a r^{-1}\left(B_{1}\right)=B$. Set $R=\Delta^{-1}(P)$. We compute

$$
\begin{aligned}
\operatorname{Darit}(P) \cdot B_{1}= & -\operatorname{dar}(\operatorname{arit}(R) \cdot B)+\operatorname{dar}([R, B]) \\
= & -u_{1} \cdots u_{r}\left(R\left(u_{1}, \ldots, u_{r-1}\right)-R\left(u_{2}, \ldots, u_{r}\right)\right) \\
& \quad-u_{1} \cdots u_{r}\left(R\left(u_{2}, \ldots, u_{r}\right)-R\left(u_{1}, \ldots, u_{r-1}\right)\right) \\
= & 0 .
\end{aligned}
$$


This concludes the proof of Lemma 3.1.1.

We consider by default that $a$ is alternal and polynomial. Let $\left(A R I_{l u}^{a}\right)^{p o l, a l}$ denote the Lie subalgebra of alternal polynomial moulds of $A R I_{l u}^{a}$. Then $A R I_{l u}^{p o l, a l}$ is a Lie ideal of $A R I_{l u}^{a}$ and we have the Lie algebra isomorphism

$$
L[C] \rtimes \mathbb{Q} a \simeq \operatorname{Lie}[a, b] \stackrel{m a}{\longrightarrow}\left(A R I_{l u}^{a}\right)^{p o l, a l} \simeq A R I_{l u}^{p o l, a l} \rtimes \mathbb{Q} a .
$$

Lemma 3.1.2. Suppose that $P \in A R I$ is a mould such that Darit $(P)$ preserves the Lie subalgebra $\left(A R I_{l u}^{a}\right)^{\text {pol,al }}$ of $A R I_{l u}^{a}$. Then there exists a derivation $E_{P} \in \operatorname{Der} \operatorname{Lie}[a, b]$ that corresponds to $\operatorname{Darit}(P)$ restricted to $\left(A R I_{l u}^{a}\right)^{\text {pol,al }}$, in the sense that

$$
m a\left(E_{P}(f)\right)=\operatorname{Darit}(P)(m a(f)) \quad \text { for all } f \in \operatorname{Lie}[a, b] .
$$

The derivation $E_{P}$ has the property that the values $E_{P}(a)$ and $E_{P}(b)$ lie in $\operatorname{Lie}[C]$.

Proof. By the isomorphism (3.1.4), every mould $P \in\left(A R I_{l u}^{a}\right)^{p o l, a l}$ has a unique preimage in Lie $[a, b]$ under $m a$ : we write $p=m a^{-1}(P)$. Recall that $B=m a(b)$. By assumption, $P$ is an alternal polynomial-valued mould, and so is $\operatorname{Darit}(P) \cdot B$ since $P$ preserves such moulds. Thus we can define $E_{P}$ by setting $E_{P}(a)=m a^{-1}(P), E_{P}(b)=m a^{-1}(\operatorname{Darit}(P) \cdot B)$. In particular this means that the monomial $a$ does not appear in the polynomials $E_{P}(a)$ and $E_{P}(b)$.

Lemma 3.1.3. Let $P$ be an alternal polynomial-valued mould. Then Darit $(P)$ preserves $\left(A R I_{l u}^{a}\right)^{\text {pol,al }}$ if and only if $P$ is push-invariant.

Proof. By the isomorphism (3.1.4), $\left(A R I_{l u}^{a}\right)^{p o l, a l}$ is generated as a Lie algebra under the $l u$ bracket by $m a(a)=a$ and $m a(b)=B$. Since $\operatorname{Darit}(P) \cdot a=P$ is alternal and polynomial-valued by assumption, it suffices to determine when $\operatorname{Darit}(P) \cdot B$ is alternal and polynomial. Let $N=\Delta^{-1} P$, and set $B_{-1}=d a r^{-1}(B)$, so $B_{-1}$ is concentrated in depth 1 with $B_{-1}\left(u_{1}\right)=1 / u_{1}$. We compute

$$
\begin{gathered}
(\operatorname{Darit}(P) \cdot B)\left(u_{1}, \ldots, u_{r}\right)=-\operatorname{dar}\left(\operatorname{arit}(N) \cdot B_{-1}-\left[N, B_{-1}\right]\right)\left(u_{1}, \ldots, u_{r}\right) \\
=-\operatorname{dar}\left(\operatorname{arit}(N) \cdot B_{-1}\right)\left(u_{1}, \ldots, u_{r}\right)-\operatorname{dar}\left(\left[B_{-1}, N\right]\right)\left(u_{1}, \ldots, u_{r}\right) \\
=-\operatorname{dar}\left(B_{-1}\left(u_{1}+\cdots+u_{r}\right)\left(N\left(u_{1}, \ldots, u_{r-1}\right)-N\left(u_{2}, \ldots, u_{r}\right)\right)\right) \\
\quad-u_{1} \ldots u_{r}\left(B_{-1}\left(u_{1}\right) N\left(u_{2}, \ldots, u_{r}\right)+N\left(u_{1}, \ldots, u_{r-1}\right) B_{-1}\left(u_{r}\right)\right) \\
=-u_{1} \cdots u_{r}\left(u_{1}+\cdots+u_{r}\right)^{-1}\left(N\left(u_{1}, \ldots, u_{r-1}\right)-N\left(u_{2}, \ldots, u_{r}\right)\right) \\
\quad-u_{2} \cdots u_{r} N\left(u_{2}, \ldots, u_{r}\right)+u_{1} \cdots u_{r-1} N\left(u_{1}, \ldots, u_{r-1}\right) \\
=\frac{1}{u_{1}+\cdots+u_{r}}\left(P\left(u_{1}, \ldots, u_{r-1}\right)-P\left(u_{2}, \ldots, u_{r}\right)\right) .
\end{gathered}
$$

In order for this mould to be polynomial-valued, it is necessary and sufficient that the numerator should be zero when $u_{r}=-u_{1}-\cdots-u_{r-1}$, i.e., that

$$
P\left(u_{1}, \ldots, u_{r-1}\right)=P\left(u_{2}, \ldots, u_{r-1},-u_{1}-\cdots-u_{r-1}\right) .
$$

But the right-hand term is equal to $\mathrm{push}^{-1}(P)$, so this condition is equivalent to the push-invariance of $P$.

Corollary 3.1.4. The derivation $E_{\psi}$ defined in section 2.2 is equal to the derivation $E_{M}$ associated to Darit $(M)$ as in Lemma 3.1.2. 
Proof. Since $M$ is push-invariant by Corollary 1.3.4, Darit $(M)$ preserves $\left(A R I_{l u}^{a}\right)^{\text {pol,al }}$ by Lemma 3.1.3. Thus we are in the situation of Lemma 3.1.2, so there exists a derivation $E_{M}$ of Lie $[a, b]$ such that $E_{M}(a)=m$ with $m a(m)=M$. Furthermore, setting $B_{1}=m a([b, a])$, we know that $\operatorname{Darit}(M) \cdot B_{1}=0$ by Lemma 3.1.1 (iv), and therefore by Lemma 3.1.2, we have $E_{M}([b, a])=$ $E_{M}([a, b])=0$. Thus the derivation $E_{M}$ of $\operatorname{Lie}[a, b]$ agrees with $E_{\psi}$ on $a$ and on $[a, b]$, so since furthermore $E_{M}(b) \in \operatorname{Lie}[a, b] \ominus \operatorname{Lie}[a]$, they are equal.

This result means that we can now use mould theoretic methods to study $\operatorname{Darit}(M)$ in order to prove Proposition 2.2.1.

\subsection{The $\Delta$-operator}

Let us define a new Lie bracket, the Dari-bracket, on $A R I$ by

$$
\operatorname{Dari}(P, Q)=\operatorname{Darit}(P) \cdot Q-\operatorname{Darit}(Q) \cdot P,
$$

where $\operatorname{Darit}(P)$ is the $l u$-derivation defined in (3.1.1). Let $A R I_{\text {Dari }}$ denote the Lie algebra obtained by equipping $A R I$ with this Lie bracket.

Proposition 3.2.1. The operator $\Delta$ is a Lie algebra isomorphism from $A R I_{\text {ari }}$ to $A R I_{\text {Dari }}$.

Proof. Certainly $\Delta$ is a vector space isomorphism from $A R I_{\text {ari }}$ to $A R I_{\text {Dari }}$ since it is an invertible operator on moulds. To prove that it is a Lie algebra isomorphism, we need to show the Lie bracket identity $\Delta(\operatorname{ari}(P, Q))=\operatorname{Dari}(\Delta P, \Delta Q)$, or equivalently,

$$
\operatorname{Dari}(P, Q)=\Delta\left(\operatorname{ari}\left(\Delta^{-1} P, \Delta^{-1} Q\right)\right)
$$

for all moulds $P, Q \in A R I$. But indeed, we have

$$
\begin{aligned}
& \operatorname{Dari}(P, Q)=\operatorname{Darit}(P) \cdot Q-\operatorname{Darit}(Q) \cdot P \\
& =-\left(\operatorname{dar} \circ \operatorname{arit}\left(\Delta^{-1} P\right) \circ \operatorname{dar}^{-1}\right) \cdot Q+\left(\operatorname{dar} \circ \operatorname{ad}\left(\Delta^{-1} P\right) \circ \operatorname{dar}^{-1}\right) \cdot Q \\
& +\left(\text { dar } \circ \operatorname{arit}\left(\Delta^{-1} Q\right) \circ \operatorname{dar}^{-1}\right) \cdot P-\left(\operatorname{dar} \circ \operatorname{ad}\left(\Delta^{-1} Q\right) \circ d a r^{-1}\right) \cdot P \\
& =-\left(\Delta \circ \operatorname{arit}\left(\Delta^{-1} P\right) \circ \Delta^{-1}\right) \cdot Q+\left(\Delta \circ \operatorname{arit}\left(\Delta^{-1} Q\right) \circ \Delta^{-1}\right) \cdot P \\
& +\left(\text { dar } \circ \operatorname{ad}\left(\Delta^{-1} P\right) \circ d a r^{-1}\right) \cdot Q-\left(\text { dar } \circ \operatorname{ad}\left(\Delta^{-1} Q\right) \circ d a r^{-1}\right) \cdot P \\
& =-\left(\Delta \circ \operatorname{arit}\left(\Delta^{-1} P\right) \circ \Delta^{-1}\right) \cdot Q+\left(\Delta \circ \operatorname{arit}\left(\Delta^{-1} Q\right) \circ \Delta^{-1}\right) \cdot P \\
& +\operatorname{dar}\left(\left[\Delta^{-1}(P), \operatorname{dar}^{-1} Q\right]\right)-\operatorname{dar}\left(\left[\Delta^{-1}(P), \operatorname{dar}^{-1} P\right]\right) \\
& =\Delta\left(-\operatorname{arit}\left(\Delta^{-1} P \cdot \Delta^{-1} Q+\operatorname{arit}\left(\Delta^{-1} Q\right) \cdot \Delta^{-1} P\right.\right. \\
& \left.+\operatorname{dur}^{-1}\left(\left[\Delta^{-1} P, \operatorname{dar}^{-1} Q\right]+\left[\operatorname{dar}^{-1} P, \Delta^{-1} Q\right]\right)\right) \\
& =\Delta\left(-\operatorname{arit}\left(\Delta^{-1} P \cdot \Delta^{-1} Q+\operatorname{arit}\left(\Delta^{-1} Q\right) \cdot \Delta^{-1} P\right.\right. \\
& \left.+\operatorname{dur}^{-1}\left(\left[\Delta^{-1} P, \operatorname{dur} \Delta^{-1} Q\right]+\left[\operatorname{dur} \Delta^{-1} P, \Delta^{-1} Q\right]\right)\right) \\
& =\Delta\left(-\operatorname{arit}\left(\Delta^{-1} P \cdot \Delta^{-1} Q+\operatorname{arit}\left(\Delta^{-1} Q\right) \cdot \Delta^{-1} P\right.\right. \\
& \left.+\operatorname{dur}{ }^{-1} d u r\left(\left[\Delta^{-1} P, \Delta^{-1} Q\right]\right)\right) \\
& =\Delta\left(-\operatorname{arit}\left(\Delta^{-1} P \cdot \Delta^{-1} Q+\operatorname{arit}\left(\Delta^{-1} Q\right) \cdot \Delta^{-1} P+\left[\Delta^{-1} P, \Delta^{-1} Q\right]\right)\right. \\
& =\Delta\left(\operatorname{ari}\left(\Delta^{-1} P, \Delta^{-1} Q\right)\right),
\end{aligned}
$$

which proves the desired identity. 
Let us now define the group $G A R I_{\text {Dgari }}$. We start by defining the exponential map $\exp _{\text {Dari }}$ : $A R I_{\text {Dari }} \rightarrow$ GARI by

$$
\exp _{\text {Dari }}(P)=1+\sum_{n \geq 1} \frac{1}{n !} \operatorname{Darit}(P)^{n-1}(P),
$$

which for all $P \in A R I$ satisfies the equality

$$
\exp (\operatorname{Darit}(P))(a)=\exp _{\text {Dari }}(P) .
$$

This map is easily seen to be invertible, since for any $Q \in G A R I$ we can recover $P$ such that $\exp _{\text {Dari }}(P)=Q$ recursively depth by depth. Let $\log _{\text {Dari }}$ denote the inverse of $\exp _{\text {Dari }}$. For each $P \in G A R I$, we then define an automorphism $\operatorname{Dgarit}(P) \in$ Aut $A R I_{l u}$ by

$$
\operatorname{Dgarit}(P)=\operatorname{Dgarit}\left(\exp _{\text {Dari }}\left(\log _{\text {Dari }}(P)\right)\right)=\exp \left(\operatorname{Darit}\left(\log _{\text {Dari }}(P)\right)\right) \text {. }
$$

Finally, we define the multiplication Dgari on GARI by

$$
\begin{aligned}
\operatorname{Dgari}(P, Q) & =\exp _{\text {Dari }}\left(\operatorname{ch}_{\text {Dari }}\left(\log _{\text {Dari }}(P), \log _{\text {Dari }}(Q)\right)\right) \\
& =\exp \left(\operatorname{Darit}\left(\log _{\text {Dari }}(P)\right)\right) \circ \exp \left(\operatorname{Darit}\left(\log _{\text {Dari }}(Q)\right)\right) \cdot a \\
& =\operatorname{Dgarit}(P) \circ \operatorname{Dgarit}(Q) \cdot a \\
& =\operatorname{Dgarit}(P) \cdot Q
\end{aligned}
$$

where $c h_{\text {Dari }}$ denotes the Campbell-Hausdorff law on $A R I_{\text {Dari }}$. We obtain the following commutative diagram, analogous to Ecalle's diagram (A.18) (cf. Appendix):

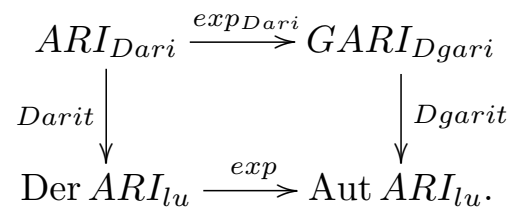

Lemma 3.2.2. For any mould $P \in G A R I$, the automorphism Dgarit $(P)$ of $A R I_{l u}$ extends to an automorphism of the Lie algebra ARI $I_{\text {lu }}^{a}$ with the following properties:

i) its value on a is given by

$$
\operatorname{Dgarit}(P) \cdot a=a-1+P \in A R I^{a} ;
$$

ii) we have Dgarit $(P) \cdot B_{1}=B_{1}$.

Proof. Let $Q=\log _{\text {Dari }}(P) \in A R I$. We saw in Lemma 3.1.1 (iv) that $\operatorname{Darit}(Q)$ extends to $A R I_{l u}^{a}$ with $\operatorname{Darit}(Q) \cdot a=Q$. By diagram (3.2.4), we have

$$
\begin{aligned}
\operatorname{Dgarit}(P) \cdot a & =\operatorname{Dgarit}\left(\exp _{\text {Dari }}(Q)\right) \cdot a \\
& =\exp (\operatorname{Darit}(Q)) \cdot a \\
& =a+\operatorname{Darit}(Q) \cdot a+\frac{1}{2} \operatorname{Darit}(Q)^{2} \cdot a+\cdots \\
& =a+Q+\frac{1}{2} \operatorname{Darit}(Q) \cdot Q+\cdots \\
& =a-1+\exp _{\text {Dari }}(Q) \text { by }(3.2 .2) \\
& =a-1+P .
\end{aligned}
$$


The second statement follows immediately from the fact that $\operatorname{Darit}(Q) \cdot B_{1}=0$ for all $Q \in A R I$ shown in Lemma 3.1.1 (iv).

Finally, we set $\Delta^{*}=e x p_{D a r i} \circ \Delta \circ \log _{a r i}$, to obtain the commutative diagram of isomorphisms

$$
\begin{array}{cc}
A R I_{\text {ari }} \stackrel{\Delta}{\longrightarrow} & A R I_{\text {Dari }} \\
\exp _{\text {ari }} \downarrow & \downarrow \exp _{\text {Dari }} \\
G A R I_{\text {gari }} \stackrel{\Delta^{*}}{\rightarrow} & A R I_{\text {Dgari }},
\end{array}
$$

which will play a special role in the proof of Proposition 2.2.1. Indeed, the key result in our proof Proposition 2.2.1 is an explicit formula for the map $\Delta^{*}$. In order to formulate it, we first define the mu-dilator of a mould, introduced by Ecalle in [E2].

Definition. Let $P \in G A R I$. Then the $m u$-dilator of $P$, denoted $d u P$, is defined by

$$
d u P=P^{-1} d u r(P) .
$$

Ecalle writes this in the equivalent form $\operatorname{dur}(P)=P d u P$, and by (3.1.3), this means that $[P, a]=$ $P a-a P=P d u P=P$, whch multiplying by $P^{-1}$, gives us the useful formulation ${ }^{6}$

$$
P^{-1} a P=a-d u P .
$$

Proposition 3.2.3. The isomorphism

$$
\Delta^{*}: G A R I_{\text {gari }} \rightarrow G A R I_{\text {Dgari }}
$$

in diagram (3.2.6) is explicitly given by the formula

$$
\Delta^{*}(Q)=1-\operatorname{dar}\left(\text { duinv }_{\text {gari }}(Q)\right) .
$$

Proof. Let $Q \in G A R I$, and set $P=\log _{\text {ari }}(Q)$. Let $R=\exp _{\text {ari }}(-P)$. By Lemma A.1 from the Appendix, the derivation $-\operatorname{arit}(P)+\operatorname{ad}(P)$ extends to $a$ taking the value $[a, P]$ on $a$, and we have

$$
\exp (-\operatorname{arit}(P)+\operatorname{ad}(P)) \cdot a=R^{-1} a R \text {. }
$$

By (3.1.1), we have

$$
\exp (\operatorname{Darit}(\Delta(P)))=\operatorname{dar} \circ \exp (-\operatorname{arit}(P)+\operatorname{ad}(P)) \circ \operatorname{dar}^{-1} .
$$

Recall that $\operatorname{dar}(a)=a$ by Lemma 3.1.1 (i), and $d a r$ is an automorphism of $A R I_{l u}^{a}$; in particular $d u$ commutes with dar. Thus we have

$$
\begin{aligned}
\exp (\operatorname{Darit}(\Delta(P))) \cdot a & =\operatorname{dar} \circ \exp (-\operatorname{arit}(P)+\operatorname{ad}(P)) \cdot a \\
& =\operatorname{dar}\left(R^{-1} a R\right) \quad \text { by Lemma A.1 } \\
& =\operatorname{dar}(R)^{-1} a \operatorname{dar}(R) \\
& =a-d u(\operatorname{dar}(R)) \text { by }(3.2 .8) \\
& =a-\operatorname{dar}(d u R) .
\end{aligned}
$$

${ }^{6}$ We are grateful to B. Enriquez for spotting this enlightening interpretation of the $m u$-dilator, which cannot even be stated meaningfully for general moulds unless $a$ is added to ARI. 
Now, using $P=\log _{\text {ari }}(Q)$, we compute

$$
\begin{aligned}
\Delta^{*}(Q) & =1-a+D \operatorname{garit}\left(\Delta^{*}(Q)\right) \cdot a \text { by }(3.2 .5) \\
& =1-a+D \operatorname{garit}\left(\exp _{\text {Dari }}\left(\Delta\left(\log _{\text {ari }}(Q)\right)\right)\right) \cdot a \text { by }(3.2 .6) \\
& =1-a+\operatorname{Dgarit}\left(\exp _{\text {Dari }}(\Delta(P))\right) \cdot a \\
& \left.=1-a+\exp \left(\operatorname{Darit}_{(\Delta)}(P)\right)\right) \cdot a \text { by }(3.2 .4) \\
& =1-d \operatorname{dar}\left(\operatorname{duexp}_{\text {ari }}(-P)\right) \text { by }(3.2 .11) \\
& =1-d \operatorname{dar}\left(\text { duinv }_{\text {gari }}(Q)\right) .
\end{aligned}
$$

This proves the proposition.

Corollary. We have the identity

$$
\Delta^{*}(\text { invpal })=m a\left(1-a+\operatorname{Ber}_{-b}(a)\right) .
$$

Proof. Applying (3.2.9) to $Q=i n v p a l=i n v_{\text {gari }}($ pal $)$, we find

$$
\Delta^{*}(\text { invpal })=1-\operatorname{dar}(\text { dupal })
$$

where dupal is the mu-dilator of pal given in (1.3.3), discovered by Ecalle. Comparing the elementary mould identity

$$
m a\left(a d(-b)^{r}(-a)\right)=\sum_{j=0}^{r-1}(-1)^{j}\left(\begin{array}{c}
r-1 \\
j
\end{array}\right) u_{j+1}
$$

with (1.3.3) shows that dar(dupal) is given in depth $r \geq 1$ by

$$
\operatorname{dar}(\text { dupal })\left(u_{1}, \ldots, u_{r}\right)=\frac{B_{r}}{r !} \sum_{j=0}^{r-1}(-1)^{j}\left(\begin{array}{c}
r-1 \\
j
\end{array}\right) u_{j+1}=\frac{B_{r}}{r !} m a\left(a d(-b)^{r}(-a)\right) .
$$

Since the constant term of $\operatorname{dar}(d u p a l)(\emptyset)$ is 0 , this yields

$$
d a r(d u p a l)=m a\left(\operatorname{Ber}_{-b}(-a)+a\right)=m a\left(a-\operatorname{Ber}_{-b}(a)\right)
$$

so (3.2.14) implies the desired identity (3.2.13).

\subsection{Proof of Proposition 2.2.1}

Let $\psi \in \mathfrak{g r t}$. We return to the notation of (1.3.8). By Corollary 3.1.4, we have a derivation $E_{M}=E_{\psi} \in \operatorname{Der} \operatorname{Lie}[a, b]$ obtained by restricting the derivation $\mathcal{E}_{\psi}=\operatorname{Darit}(M)$ to the Lie subalgebra of $A R I_{l u}^{a}$ generated by $a$ and $B=m a(b)$, which is precisely $\left(A R I_{l u}^{a}\right)^{p o l, a l}$, and transporting the derivation to the isomorphic space Lie $[a, b]$. The purpose of this section is to prove (2.2.1), i.e.,

$$
E_{\psi}\left(t_{02}\right)=\left[\psi\left(t_{02}, t_{12}\right), t_{02}\right] .
$$

The main point is the following result decomposing Darit $(M)$ into three factors; a derivation conjugated by an automorphism. We note that although the values of the derivation and the 
automorphism in Proposition 3.3.1 on $a$ are polynomial-valued moulds, this is false for their values on $B=m a(b)$, which means that this decomposition is a result which cannot be stated in the power-series situation of Lie $[a, b]$; the framework of mould theory admitting denominators is crucial here.

Proposition 3.3.1. We have the following identity of derivations:

$$
\begin{gathered}
\operatorname{Darit}\left(\Delta\left(\operatorname{Ad}_{\text {ari }}(\text { invpal }) \cdot F\right)\right)= \\
\operatorname{Dgarit}\left(\Delta^{*}(\text { invpal })\right) \circ \operatorname{Darit}(\Delta(F)) \circ \operatorname{Dgarit}\left(\Delta^{*}(\text { invpal })\right)^{-1} .
\end{gathered}
$$

Proof. We use two standard facts about Lie algebras and their exponentials. Firstly, for any exponential morphism exp $: \mathfrak{g} \rightarrow G$ mapping a Lie algebra to its associated group, the natural adjoint action of $G$ on $\mathfrak{g}$, denoted $A d_{\mathfrak{g}}(\exp (g)) \cdot h$, satisfies

$$
\exp \left(A d_{\mathfrak{g}}(\exp (g)) \cdot h\right)=A d_{G}(\exp (g))(\exp (h))=\exp (g) *_{G} \exp (h) *_{G} \exp (g)^{-1},
$$

where $*_{G}$ denotes the multiplication in $G$, defined by

$$
\exp (g) *_{G} \exp (h)=\exp \left(\operatorname{ch}_{\mathfrak{g}}(g, h)\right)
$$

where $c h_{\mathfrak{g}}$ denotes the Campbell-Hausdorff law on $\mathfrak{g}$.

Secondly, if $\Delta: \mathfrak{g} \rightarrow \mathfrak{h}$ is an isomorphism of Lie algebras, then the following diagram commutes:

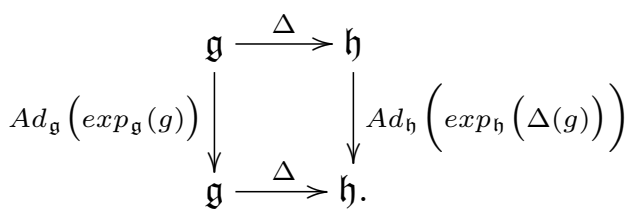

To prove (3.3.1), we start by taking the exponential of both sides. Let lipal $=\log _{\text {ari }}($ invpal $)$. We start with the left-hand side and compute

$$
\begin{aligned}
& \exp \left(\operatorname{Darit}\left(\Delta\left(A_{\text {ari }}(\text { invpal }) \cdot F\right)\right)\right)=\exp \left(\operatorname{Darit}\left(\Delta\left(\operatorname{Ad}_{\text {ari }}\left(\exp _{\text {ari }}(\operatorname{lipal})\right) \cdot F\right)\right)\right) \\
& \left.=\exp \left(\operatorname{Darit}\left(\operatorname{Ad}_{\text {Dari }}\left(\exp _{\text {Dari }}(\Delta l i p a l)\right) \cdot \Delta(F)\right)\right)\right) \\
& =\operatorname{Dgarit}\left(\exp _{\text {Dari }}\left(A d_{\text {Dari }}\left(\exp _{\text {Dari }}(\Delta l i p a l)\right) \cdot \Delta(F)\right)\right) \\
& =\operatorname{Dgarit}\left(\exp _{\text {Dari }}(\Delta \text { lipal })\right) \circ \operatorname{Dgarit}\left(\exp _{\text {Dari }}(\Delta(F))\right) \circ \operatorname{Dgarit}\left(\exp _{\text {Dari }}(\Delta \text { lipal })\right)^{-1} \\
& =\operatorname{Dgarit}\left(\Delta^{*}(\text { invpal })\right) \circ \exp (\operatorname{Darit}(\Delta(F))) \circ \operatorname{Dgarit}\left(\Delta^{*}(\text { invpal })\right)^{-1} \text {, }
\end{aligned}
$$

where the second equality follows from (3.3.4) (with $\mathfrak{g}, \exp _{\mathfrak{g}}$ and $A d_{\mathfrak{g}}$ identified with $A R I_{\text {ari }}$, $\exp _{\text {ari }}$ and $A d_{\text {ari }}$, and the same three terms for $\mathfrak{h}$ with the corresponding terms for $\left.A R I_{\text {Dari }}\right)$, the third from (3.2.4), the fourth from (3.3.2) and the fifth again from (3.2.4). But the first and last 
expressions in (3.3.5) are equal to the exponentials of the left- and right-hand sides of (3.3.1). This concludes the proof of the Proposition.

We can now complete the proof of Proposition 2.2.1 by using Proposition 3.3.1 to compute the value of $E_{\psi}\left(t_{02}\right)$. By (3.2.9) and the Corollary to Proposition 3.2.3, we have

$$
\operatorname{Dgarit}\left(\Delta^{*}(\text { invpal })\right) \cdot a=a-1+\Delta^{*}(\text { invpal })=m a\left(\operatorname{Ber}_{-b}(a)\right)=m a\left(t_{02}\right) .
$$

Recall that $E_{\psi}$ is nothing but the polynomial version of $\operatorname{Darit}(M)$ restricted to the Lie algebra generated by the moulds $a$ and $B$. Thus, to compute the value of $E_{\psi}$ on $t_{02}=\operatorname{Ber}_{-b}(a)$, we can now simply use (3.3.1) to compute the value of $\operatorname{Darit}(M)$ on $m a\left(t_{02}\right)$. By (3.3.6), the rightmost map of the right-hand side of (3.3.1) maps $m a\left(t_{02}\right)$ to $a$. By Lemma 3.1.1 (iv), the derivation $\operatorname{Darit}(P)$ for any mould $P \in A R I$ extends to $a$ taking the value $P$ on $a$, so we can apply the middle map of (3.3.1) to $a$, obtaining

$$
\begin{gathered}
\operatorname{Darit}(\Delta(F)) \cdot a=\Delta(F)=\operatorname{dur}(\operatorname{dar}(F))=m a([f(a,[b, a]), a]) \\
=m a([\psi(a,[a, b]), a])=m a\left(\left[\psi\left(a, t_{12}\right), a\right]\right) .
\end{gathered}
$$

Finally, we note that by Lemma 3.2.2 (ii), the leftmost map of the right-hand side of (3.3.1) fixes $B_{1}=-m a\left(t_{12}\right)$, so it also fixes $m a\left(t_{12}\right)$. By (3.3.6), it sends $a$ to $m a\left(t_{02}\right)$, so applying it to the rightmost term of (3.3.7) we obtain the total expression

$$
\operatorname{Darit}(M)\left(m a\left(t_{02}\right)\right)=m a\left(\left[\psi\left(t_{02}, t_{12}\right), t_{02}\right]\right) .
$$

In terms of polynomials, this gives the desired expression

$$
E_{\psi}\left(t_{02}\right)=\left[\psi\left(t_{02}, t_{12}\right), t_{02}\right],
$$

which concludes the proof. 


\section{Appendix: Mould basics}

For the purposes of this article, we use the term "mould" to refer only to rational-function valued moulds with coefficients in $\mathbb{Q}$; thus, a mould is a family of functions $\left\{P_{r}\left(u_{1}, \ldots, u_{r}\right) \mid r \geq 0\right\}$ with $P_{r}\left(u_{1}, \ldots, u_{r}\right) \in \mathbb{Q}\left(u_{1}, \ldots, u_{r}\right)$. In particular $P_{0}(\emptyset)$ is a constant. The depth $r$ part of a mould is the function $P_{r}\left(u_{1}, \ldots, u_{r}\right)$ in $r$ variables. By defining addition and scalar multiplication of moulds in the obvious way, i.e., depth by depth, moulds form a $\mathbb{Q}$-vector space that we call Moulds. Following Ecalle, we often drop the subscript $r$ from the mould notation; i.e., we write $P\left(u_{1}, \ldots, u_{r}\right)$ to mean the rational function $P_{r}\left(u_{1}, \ldots, u_{r}\right)$, where the number of variables automatically indicates which depth part we are considering.

We write $G A R I$ for the set of moulds with $P(\emptyset)=1$, and $A R I$ for the set of moulds ${ }^{8}$ with $P(\emptyset)=0$. Then $A R I$ forms a vector subspace of Moulds.

Let $(\text { Moulds })^{\text {pol }}$ denote the subspace of polynomial-valued moulds, i.e., moulds such that $P\left(u_{1}, \ldots, u_{r}\right)$ is a polynomial in each depth $r \geq 0$, and $A R I^{p o l}$ the polynomial-valued subspace of $A R I$. In this appendix we will stress the connections between polynomial-valued moulds and power series in the non-commutative variables $a$ and $b$, showing in particular how familiar notions from multizeta theory (the Poisson-Ihara bracket, the twisted Magnus group etc.) not only translate over to the corresponding moulds, but generalize to all moulds.

Let $C_{i}=a d(a)^{i-1}(b)$ for $i \geq 1$. Let the depth of a monomial $C_{i_{1}} \cdots C_{i_{r}}$ be the number $r$ of $C_{i}$ in the monomial; the depth forms a grading on the free associative ring of polynomials in the $C_{i}$ 's. Let $\mathbb{Q}\langle C\rangle=\mathbb{Q}\left\langle C_{1}, C_{2}, \ldots\right\rangle$ denote the depth completion of this ring, i.e., $\mathbb{Q}\langle C\rangle$ is the space of power series that are polynomials in each depth. We also write

$$
L[C]=\operatorname{Lie}\left[C_{1}, C_{2}, \ldots\right]
$$

for the corresponding free Lie algebra. Note that the freeness follows from Lazard elimination, which also shows that we have the isomorphism

$$
\mathbb{Q} a \oplus L[C] \simeq \operatorname{Lie}[a, b] .
$$

Ecalle uses the notation $m a$ to denote the standard vector space isomorphism from $\mathbb{Q}\langle C\rangle$ to the space (Moulds) ${ }^{\text {pol }}$ of polynomial-valued moulds defined by

$$
\begin{aligned}
& \text { ma: } \mathbb{Q}\langle C\rangle \stackrel{\sim}{\rightarrow}(\text { Moulds })^{\text {pol }} \\
& C_{k_{1}} \cdots C_{k_{r}} \mapsto(-1)^{k_{1}+\cdots+k_{r}-r} u_{1}^{k_{1}-1} \cdots u_{r}^{k_{r}-1}
\end{aligned}
$$

on monomials and extended by linearity. This map ma can also be considered as a ring isomorphism when (Moulds) ${ }^{p o l}$ is equipped with the suitable multiplication, cf. the remarks following (A.4) below. (We use the same notation $m a$ when $C_{i}=a d(x)^{i-1}(y)$, for polynomials usually considered in Lie $[x, y]$, such as polynomials in $\mathfrak{g r t}$.) For any map $\Phi: \mathbb{Q}\langle C\rangle \rightarrow \mathbb{Q}\langle C\rangle$, we define its transport $m a(\Phi)$ to $(\text { Moulds })^{\text {pol }}$, namely the corresponding map on polynomial-valued moulds

$$
m a(\Phi):(\text { Moulds })^{\text {pol }} \rightarrow(\text { Moulds })^{\text {pol }}
$$

by the obvious relation

$$
m a(\Phi)(m a(f))=m a(\Phi(f)) \text { for all } f \in \mathbb{Q}\langle C\rangle .
$$

8 Ecalle uses the notation $A R I$ for the space of these moulds equipped with the ari-bracket, that we denote $A R I_{\text {ari }}$, and in fact he considers more general bimoulds in two sets of variables. 
Power series, moulds, standard multiplication and Lie bracket. Via the map (A.2), many of the familiar notions associated with power series and Lie series pass to polynomial moulds, with general expressions that are in fact valid for all moulds.

In particular, the standard mould multiplication $m u$ is given by

$$
m u(P, Q)\left(u_{1}, \ldots, u_{r}\right)=\sum_{i=0}^{r} P\left(u_{1}, \ldots, u_{i}\right) Q\left(u_{i+1}, \ldots, u_{r}\right) .
$$

For simplicity, we write $P Q=m u(P, Q)$. The multiplication $m u$ generalizes ordinary multiplication of non-commutative power series in the sense that

$$
m a(f g)=m u(m a(f), m a(g))=m a(f) m a(g)
$$

for $f, g \in \mathbb{Q}\langle C\rangle$. The space $\left(\right.$ Moulds) ${ }^{p o l}$ is a ring under the $m u$ multiplication, generated by the depth 1 polynomial moulds $B_{i}$ given by $B_{i}\left(u_{1}\right)=u_{1}^{i}$ for $i \geq 0$. By (A.4), the linear map $m a$ from (A.2) can be defined as a ring isomorphism from $\mathbb{Q}\langle C\rangle$ to (Moulds) ${ }^{\text {pol }}$, taking values $m a\left(C_{i}\right)=(-1)^{i-1} B_{i-1}$ on the generators $C_{i}$ for $i \geq 1$.

A mould $P$ is invertible for the $m u$-multiplication if and only if its constant term $P(\emptyset) \in \mathbb{Q}$ is invertible. If the constant term is 1 , the formula for the $m u$-inverse $P^{-1}=i n v m u(P)$ is explicitly given by

$$
P^{-1}(\mathbf{u})=\sum_{0 \leq s \leq r}(-1)^{s} \sum_{\mathbf{u}=\mathbf{u}_{1} \cdots \mathbf{u}_{s}} P\left(\mathbf{u}_{1}\right) \cdots P\left(\mathbf{u}_{s}\right),
$$

where the sum runs over all ways $\mathbf{u}_{1} \cdots \mathbf{u}_{s}$ of cutting the word $\mathbf{u}=\left(u_{1}, \ldots, u_{r}\right)$ into $s$ nonempty chunks. By (A.4), if $f \in \mathbb{Q}\langle C\rangle$ is invertible (i.e., has non-zero constant term), we have $m a\left(f^{-1}\right)=P^{-1}$.

The $m u$-multiplication makes $G A R I$, the set of moulds with constant term 1 , into a group that we denote by $G A R I_{m u}$. Defining the associated $l u$-bracket by $l u(P, Q)=m u(P, Q)-m u(Q, P)$, i.e., $[P, Q]=P Q-Q P$, gives ARI the structure of a Lie algebra that we call $A R I_{l u}$.

Mould symmetries. A mould $P$ is said to be alternal if

$$
\sum_{\mathbf{u} \in \operatorname{sh}\left(\left(u_{1}, \ldots, u_{i}\right),\left(u_{i+1}, \ldots, u_{r}\right)\right)} P(\mathbf{u})=0
$$

for $1 \leq i \leq r-1$.

It is well-known that $p \in \mathbb{Q}\langle C\rangle$ satisfies the shuffle relations if and only if $p$ is a Lie polynomial, i.e., $p \in \operatorname{Lie}[C]$. The alternality property on moulds is analogous to these shuffle relations, i.e., a polynomial $p \in \mathbb{Q}\langle C\rangle$ satisfies the shuffle relations if and only if $m a(p)$ is alternal. (See $[\mathrm{S}, \S 2.3$ and Lemma 3.4.1.].) This shows that, writing $A R I^{a l}$ for the subspace of alternal moulds and $A R I^{p o l, a l}$ for the subspace of alternal polynomial-valued moulds, the map ma restricts to a Lie algebra isomorphism

$$
m a: \operatorname{Lie}[C] \stackrel{m a}{\longrightarrow} A R I_{l u}^{p o l, a l} .
$$

Let the swap operator on moulds be defined by

$$
\operatorname{swap}(A)\left(v_{1}, \ldots, v_{r}\right)=A\left(v_{r}, v_{r-1}-v_{r}, \ldots, v_{1}-v_{2}\right) .
$$

Here the use of the alphabet $v_{1}, v_{2}, \ldots$ instead of $u_{1}, \ldots, u_{r}$ is purely a convenient way to distinguish a mould from its swap. The mould $\operatorname{swap}(A)$ is alternal if it satisfies the property (A.5) in the $v_{i}$ 's. 
The space of moulds that are alternal and have a swap that is also alternal is denoted $A R I^{a l / a l}$; these moulds are said to be strictly bialternal. We particularly consider the situation where a mould is alternal and its swap differs from an alternal mould by addition of a constant-valued mould. Such moulds are called bialternal, and the space of bialternal moulds is denoted $A R I^{a l * a l}$. The space of polynomial-valued bialternal moulds is denoted $A R I^{p o l, a l * a l}$. Finally, we recall that Ecalle uses the notation of underlining the symmetry of a mould to indicate that its depth 1 part is

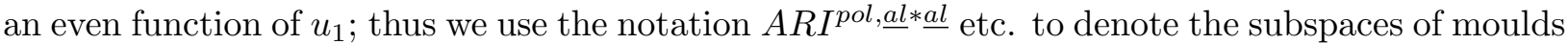
that are even in depth 1 . The subspace $A R I_{a r i}^{p o l, a l * a l}$ forms a Lie algebra under the ari-bracket (cf. $[S$, Theorem 2.5.6]), which is isomorphic under the map $m a$ to the "linearized double shuffle" Lie algebra $\mathfrak{l}$ studied for example in [Br3].

Ecalle introduces a second symmetry called alternility on moulds in the $v_{i}$ 's, which generalizes the usual stuffle relations on polynomials in $a$ and $b$. As above, we write $A R I^{a l / i l}, A R I^{a l * i l}$ and $A R I \underline{a l * i l}$ for the space of alternal moulds with swap that is alternil, resp. alternil up to addition of a constant mould, resp. also even in depth 1 . The space $A R I^{p o l, a l * \underline{i l}}$ is isomorphic under the map $m a$ to the double shuffle Lie algebra $\mathfrak{d} \mathfrak{s}$. [S, ??]

Twisted Magnus automorphism and group law. Let $G \subset \mathbb{Q}\langle C\rangle$ denote the set of power series with constant term 1 , so that $m a$ gives a bijection $G \rightarrow G A R I^{p o l}$ to the set of polynomialvalued moulds with constant term 1 . We write $\mathbf{G}$ for the group obtained by putting the standard power series multiplication on $G$, so that we have a group isomorphism $\mathbf{G} \simeq G A R I_{m u}^{p o l}$. For all $p \in G$, we define the associated "twisted Magnus" automorphism $A_{p}$ of $\mathbf{G}$, defined by $A_{p}(a)=a$, $A_{p}(b)=p b p^{-1}$. These automorphisms satisfy the composition law

$$
\left(A_{q} \circ A_{p}\right)(b)=A_{q}(p) q b q^{-1} A(p)^{-1},
$$

which defines a different multiplication on the set $G$, given by

$$
p \odot q=A_{q}(p) q=p\left(a, q b q^{-1}\right) q(a, b),
$$

satisfying

$$
A_{p \odot q}=A_{q} \circ A_{p}
$$

The inverse of the automorphism $A_{p}$ is given by $A_{q}$ where $q$ is the unique power series such that the right-hand side of (A.6) is equal to 1 . We write $G_{\odot}$ for the "twisted Magnus" group obtained by putting the multiplication law (A.6) on $G$. The association $p \mapsto A_{p}$ extends to the general case of moulds by associating to every $P \in G A R I$ the automorphism of $G A R I_{m u}$ defined by Ecalle and denoted $\operatorname{garit}(P)$, whose action on $Q \in G A R I$ is given by

$$
(\operatorname{garit}(P) \cdot Q)(\mathbf{u})=\sum_{s \geq 0} \sum_{\mathbf{u}=\mathbf{a}_{1} \mathbf{b}_{1} \mathbf{c}_{1} \cdots \mathbf{a}_{s} \mathbf{b}_{s} \mathbf{c}_{s}} Q\left(\left\lceil\mathbf{b}_{1}\right\rceil \cdots\left\lceil\mathbf{b}_{2}\right\rceil\right) P\left(\mathbf{a}_{1}\right) \cdots P\left(\mathbf{a}_{s}\right) P^{-1}\left(\mathbf{c}_{1}\right) \cdots P^{-1}\left(\mathbf{c}_{s}\right),
$$

where the sum runs over all ways of cutting the word $\mathbf{u}=\left(u_{1}, \ldots, u_{r}\right)$ into $3 s$ chunks of which the $\mathbf{b}_{i}$ 's may not be empty, $\mathbf{a}_{1}$ and $\mathbf{c}_{s}$ may be empty, and the interior chunks $\mathbf{a}_{i}$ and $\mathbf{c}_{j}$ may be empty as long as no interior double chunk $\mathbf{c}_{i} \mathbf{a}_{i+1}$ is empty. Note that because $G A R I_{m u}$ is a huge group containing all possible moulds with constant term 1, the automorphism $\operatorname{garit}(P)$ cannot be determined simply by giving its value on some simple generators as we do for $A_{p}$. However, $\operatorname{garit}(P)$ extends to $a$ taking the value $a$, and restricted to the Lie algebra $\left(A R I_{l u}^{a}\right)^{\text {pol }}$ generated by $a$ and $B$ (isomorphic to $\operatorname{Lie}[a, b]$ ), we find

$$
\operatorname{garit}(P) \cdot a=a, \quad \operatorname{garit}(P) \cdot B=P B P^{-1} .
$$


In analogy with the formula for $\odot$ given in (A.6), garit defines a multiplication law gari on GARI by the formula

$$
\operatorname{gari}(P, Q)=m u(\operatorname{garit}(Q) \cdot P, Q)=(\operatorname{garit}(Q) \cdot P) Q .
$$

We write $G A R I_{\text {gari }}$ for the group obtained by equipping $G A R I$ with this multiplication.

Poisson-Ihara bracket, exponential, linearization. For all $P \in A R I$, Ecalle defines a derivation $\operatorname{arit}(P)$ of $A R I_{l u}$ by the formula

$$
\operatorname{arit}(F) \cdot M(\mathbf{u})=\sum_{\mathbf{u}=\mathbf{a b c}, \mathbf{c} \neq \emptyset} M\left(\mathbf{a}\lceil\mathbf{c}) F(\mathbf{b})-\sum_{\mathbf{u}=\mathbf{a b c}, \mathbf{a} \neq \emptyset} M(\mathbf{a}\rceil \mathbf{c}\right) F(\mathbf{b}) .
$$

For $B=m a(b)$, i.e., $B\left(u_{1}\right)=1$, this formula yields

$$
\operatorname{arit}(P) \cdot B=[P, B]
$$

If $P=m a(f)$ for a polynomial $f \in \operatorname{Lie}[C]$, then $\operatorname{arit}(P)$ restricts to $A R I_{l u}^{p o l, a l}$, and as we saw in Lemma 3.1.1 (iii), it extends to all of $\left(A R I_{l u}^{a}\right)^{p o l, a l}$ taking the value 0 on $a$. It corresponds on the isomorphic Lie algebra Lie $[a, b]$ to the Ihara derivation $D_{f}$ defined by

$$
D_{f}(a)=0, \quad D_{f}(b)=[f, b] .
$$

The Lie bracket $\{\cdot, \cdot\}$ that we put on $L[C]$, known as the Poisson bracket or Ihara bracket, comes from bracketing the derivations $D_{f}$, i.e.,

$$
\left[D_{f}, D_{g}\right]=D_{\{f, g\}} \text { where }\{f, g\}=D_{f}(g)-D_{g}(f)-[f, g] .
$$

We obtain a pre-Lie law by linearizing the multiplication law $\odot$ defined in (A.6). In fact, because $\odot$ is linear in $p$, we only need to linearize $q$, so we write $q=1+t f$ and compute the coefficient of $t$ in

$$
p(a,(1+t f) b(1-t f))(1+t f(a, b))=p(a, b+t[f, b])(1+t f(a, b)),
$$

obtaining the expression

$$
p \odot f=p f+D_{f}(p),
$$

valid for all $p \in \mathbb{Q}\langle C\rangle, f \in L[C]$. In particular, the pre-Lie law gives another, equivalent way to obtain the Poisson bracket, namely $\{p, q\}=p \odot q-q \odot p$. The exponential map $\exp _{\odot}: L[C]_{\{\cdot, \cdot\}} \rightarrow$ $G_{\odot}$ is then defined via the pre-Lie law by

$$
\exp _{\odot}(f)=\sum_{n \geq 0} \frac{1}{n !} f^{\odot n}
$$

where the pre-Lie law is composed from left to right, so that the rightmost argument is always $f \in L[C]$. The exponential map defined this way satisfies the basic identities

$$
\exp \left(D_{f}\right)=A_{\exp _{\odot}(f)},
$$

and

$$
\exp \left(D_{f}\right) \circ \exp \left(D_{g}\right)=\exp \left(\operatorname{ch}_{\{\cdot, \cdot\}}\left(D_{f}, D_{g}\right)\right),
$$


where $c h_{\{\cdot, \cdot\}}$ denotes the Campbell-Hausdorff law on $L[C]$ equipped with the Poisson-Ihara Lie bracket (A.10).

All these standard constructions extend to the case of general moulds; Ecalle gives explicit formulas for the pre-Lie law preari and for the exponential expari, namely

$$
\operatorname{preari}(P, Q)=P Q+\operatorname{arit}(Q) \cdot P \text { and } \exp _{\text {ari }}(P)=\sum_{n \geq 0} \frac{1}{n !} \operatorname{preari}(\underbrace{P, \ldots, P}_{n}),
$$

which clearly extend (A.11) and (A.12) above, and satisfy the analogous formulas generalizing (A.13) and (A.14), namely

$$
\exp (\operatorname{arit}(P))=\operatorname{garit}\left(\exp _{\text {ari }}(P)\right)
$$

and

$$
\exp (\operatorname{arit}(P)) \circ \exp (\operatorname{arit}(Q))=\exp (\operatorname{ch}(\operatorname{arit}(P), \operatorname{arit}(Q))) .
$$

The exponential maps satisfy the properties

$$
\exp (\operatorname{arit}(P)) \circ \exp (\operatorname{arit}(Q))=\exp \left(\operatorname{arit}\left(\operatorname{ch} h_{\text {ari }}(P, Q)\right)\right)
$$

for the Campbell-Hausdorff law $c h_{\text {ari }}$ on $A R I_{a r i}$. These properties are expressed by the commutative diagram

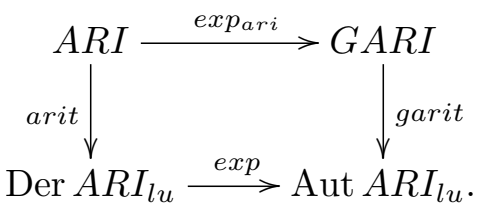

We conclude this appendix with a linearization lemma used in the proof of Proposition 3.2.3.

Lemma A.1. Let $P \in A R I$. Then the derivation - arit $(P)+\operatorname{ad}(P)$ extends to a taking the value $[P, a]$ on $a$, and we have

$$
\exp (-\operatorname{arit}(P)+a d(P)) \cdot a=R^{-1} a R
$$

where $R=\exp _{\text {ari }}(-P)$.

Proof. Since $\operatorname{arit}(P)$ extends to $a$ taking the value 0 by Lemma 3.1.1 (iii), it suffices to check that $a d(P)$ extends to $a$ via $a d(P) \cdot a=[P, a]$, i.e., that this action respects the formula $[Q, a]=d u r(Q)$. Indeed, we have

$a d(P) \cdot[Q, a]=[a d(P) \cdot Q, a]+[Q, a d(P) \cdot a]=[[P, Q], a]+[Q,[P, a]]=[P,[Q, a]]=a d(P) \cdot \operatorname{dur}(Q)$.

For a real parameter $t \in[0,1]$, let $R_{t}=\exp _{\text {ari }}(-t P)$, and let $A_{t}$ denote the automorphism of $\left(A R I_{l u}^{a}\right)^{p o l}$ defined by

$$
A_{t}(a)=R_{t}^{-1} a R_{t}, \quad A_{t}(B)=B,
$$

so that $A_{1}(a)=R^{-1} a R$. Let $D=\log (A)$; we will prove that $D=-\operatorname{arit}(P)+\operatorname{ad}(P)$ on $\left(A R I_{l u}^{a}\right)^{p o l}$. We compute $D(a)$ and $D(b)$ by the linearization formula

$$
D(a)=\left.\frac{d}{d t}\right|_{t=0}\left(A_{t}(a)\right) \quad \text { and } \quad D(b)=\left.\frac{d}{d t}\right|_{t=0}\left(A_{t}(b)\right)
$$


The second equality yields $D(b)=0$. Let us compute $D(a)$. Using $R_{0}=1$ and $\left.\frac{d}{d t}\right|_{t=0} R_{t}=-P$, we find

$$
\begin{aligned}
D(a) & =\left.\frac{d}{d t}\right|_{t=0}\left(A_{t}(a)\right) \\
& =\left.\frac{d}{d t}\right|_{t=0}\left(R_{t}^{-1} a R_{t}\right) \\
& =\left.\left(-R_{t}^{-1} \frac{d}{d t}\left(R_{t}\right) R_{t}^{-1} a R_{t}+R_{t}^{-1} a \frac{d}{d t}\left(R_{t}\right)\right)\right|_{t=0} \\
& =P a-a P .
\end{aligned}
$$

Thus $D(a)=[P, a]=(-\operatorname{arit}(P)+\operatorname{ad}(P)) \cdot a$ and $D(b)=0=(-\operatorname{arit}(P)+\operatorname{ad}(P)) \cdot b$, which concludes the proof.

\section{References}

[B] S. Baumard, Aspects modulaires et elliptiques des relations entre multizêtas, Doctoral thesis, 2014.

[BS] S. Baumard, L. Schneps, On the derivation representation of the fundamental Lie algebra of mixed elliptic motives, to appear in Annales Mathématiques du Québec, 2016.

[Br1] F. Brown, Mixed Tate motives over $\mathbb{Z}$, Ann. of Math. (2) 175, no. 2 (2012), 949-976.

[Br2] F. Brown, Talk on Anatomy of Associators, online lecture notes, http://www.ihes.fr/ brown, 2013.

[Br3] F. Brown, Zeta elements in depth 3 and the fundamental Lie algebra of a punctured elliptic curve, arXiv:1504.04737, 2015.

[Br4] F. Brown, Depth-graded motivic multiple zeta values, arXiv:1301.3053, 2013.

[E1] J. Ecalle, The flexion structure of dimorphy: flexion units, singulators, generators, and the enumeration of multizeta irreducibles, in Asymptotics in Dynamics, Geometry and PDEs; Generalized Borel Summation II, O. Costin, F. Fauvet, F. Menous, D. Sauzin, eds., Edizioni della Normale, Pisa, 2011.

[E2] J. Ecalle, Eupolars and their bialternality grid, Acta Math. Vietnam. 40 no. 4 (2015), 545-636.

[CEE] D. Calaque, B. Enriquez, P. Etingof, Universal KZB equations: the elliptic case, Algebra, Arithmetic and Geometry: in honor of Yu. I. Manin, Vol. I, 165-266, Progr. Math. 269, Birkhäuser Boston, 2009.

[En1] B. Enriquez, Elliptic Associators, Selecta Math. 20 no. 2 (2014), 491-584.

[En2] B. Enriquez, Analogues elliptiques des nombres multizetas, arXiv:1301.3042v2, to appear in Bull. Soc. Math. France, 2016.

[F] H. Furusho, Double shuffle relation for associators, Ann. of Math. 174 no. 1 (2011), 341-360.

[G] A. Goncharov, Galois symmetries of fundamental groupoids and noncommutative geometry, Duke Math J. 128 (2005) no. 2, 209-284.

[HM] R. Hain, M. Matsumoto, Universal mixed elliptic motives, arXiv:1512.03975, 2015.

[LMS] P. Lochak, N. Matthes, L. Schneps, On elliptic multiple zeta values, in preparation, 2016.

[NTU] H. Nakamura, H. Tsunogai, R. Ueno, Some stability properties of Teichmüller modular function fields with pro- $\ell$ weight structures, Math. Ann. 302, 197-213 (1995). 
[P] A. Pollack, Relations between derivations arising from modular forms, Duke University Senior Thesis, 2009.

[R] G. Racinet, Séries génératrices non commutatives de polyzêtas et associateurs de Drinfel'd, Doctoral thesis, 2000.

[S] L. Schneps, ARI, GARI, Zig and Zag, An Introduction to Ecalle's theory of moulds, arXiv:1507.01534, 2015.

[S2] L. Schneps, Double shuffle and Kashiwara-Vergne Lie algebras, J. Algebra 367 (2012), 54-74.

[So] I. Soudères, Motivic double shuffle, Int. J. Number Theory 6 (2010) no. 2, 339-370.

[T1] H. Tsunogai, On some derivations of Lie algebras related to Galois representations, Publ. RIMS 31 No. 1 (1995), 113-134.

[T2] H. Tsunogai, The stable derivation algebras for higher genera, Israel J. Math. 136 (2003), 221-250. 